\title{
Beam breakup current limit in multiturn energy recovery linear accelerators
}

\author{
W. Lou ${ }^{*}$ and G. H. Hoffstaetter \\ CLASSE, Cornell University, Ithaca, New York 14853, USA
}

(Received 24 December 2018; published 5 November 2019)

\begin{abstract}
CBETA, the Cornell BNL Energy-Recovery-Linac (ERL) Test Accelerator, is the first multiturn ERL with superconducting radio frequency (SRF) cavities cavities and will therefore be the first ERL whose current could be limited by the beam break-up (BBU) instability. To investigate the threshold current, we calculate HOMs in CBETA's cavities, including their construction errors, and simulate the BBU threshold current with the established simulation code BMAD. For some construction errors, this current limit is not sufficiently large and means of increasing it are investigated. We find that some means that have been shown effective in single turn ERLs are not practical for multiturn ERLs. To make sure that simulations are trustworthy for CBETA, we revisit the BBU theory for multiturn configurations, and compare to simulation results. We also further explore the scaling law of the BBU threshold current for the case with symmetric ERLs, and a new scaling factor has been found. BBU suppression with lattice chromaticity has been investigated assuming bunches with a Gaussian energy spread. The ability to simulate BBU effect with multiple particles per bunch has been added into BMAD, and agreement with the derived theoretical formula is found. CBETA is currently under beam commissioning at Cornell University. Our simulations show that an optimized optics will push the BBU threshold current beyond the design current, for all realistic cavity production errors.
\end{abstract}

DOI: $10.1103 /$ PhysRevAccelBeams.22.112801

\section{INTRODUCTION}

Energy recovery linacs (ERLs) open up a new regime of beam parameters with large current and simultaneously small emittances, bunch lengths, and energy spread. The Cornell BNL ERL Test Accelerator (CBETA) is the first accelerator that is constructed to analyze the potential of multipass ERLs with superconducting SRF accelerating cavities [1]. New beam parameters of ERLs allow for new experiments such as nuclear and high energy colliders, electron coolers, internal scattering experiments, x-ray sources or Compton backscattering sources for nuclear or x-ray physics [2-4]. By recirculating charged beams back into the accelerating cavities, energy can be recovered from the beams to the electromagnetic fields of the cavities. Energy recovery allows an ERL to operate at a much higher current than conventional linacs, where the current is limited by the power consumption by the cavities. While electron beams recirculate for thousands of turns in storage rings, they travel only a few turns in an ERL before being dumped. The short circulation time allows beam emittances

*w1528@cornell.edu

Published by the American Physical Society under the terms of the Creative Commons Attribution 4.0 International license. Further distribution of this work must maintain attribution to the author(s) and the published article's title, journal citation, and DOI. to be as small as for a linac. The potentials for high beam current with simultaneously low emittances allows an ERL to deliver unprecedented beam parameters.

CBETA is currently under construction at Cornell University's Wilson Laboratory. This is a collaboration with BNL, and will be the first multipass ERL with a fixed field alternating (FFA) lattice. It serves as a prototype accelerator for electron coolers of electron ion colliders (EICs). Both EIC projects in the US, eRHIC at BNL and JLEIC at TJNAF will benefit from this new accelerator [5]. Figure 1 shows the design layout of CBETA. At full operation, CBETA will be 4-pass ERL with maximum electron beam energy of $150 \mathrm{MeV}$. This is achieved by first accelerating the electron beam to $6 \mathrm{MeV}$ by the injector (IN). The beam is then accelerated by the Main Linac Cryomodule (MLC) cavities (LA) four times to reach $150 \mathrm{MeV}$, then the beam is decelerated four times down to $6 \mathrm{MeV}$ before stopped (BS). The beam passes through the MLC cavities for a total of eight times, each time with an energy gain of $\pm 36 \mathrm{MeV}$. The field energy in the cavities is transferred to the beam during acceleration, and recovered during deceleration. Transition from acceleration to deceleration is achieved by adjusting the path-length of the forth recirculation turn to be an odd multiple of half of the rf wavelength. The path-length of all the other turns is exactly an integer multiple of the rf wavelength. CBETA can also operate as a 3-pass, 2-pass, or 1-pass ERL with properly adjusted configuration. 


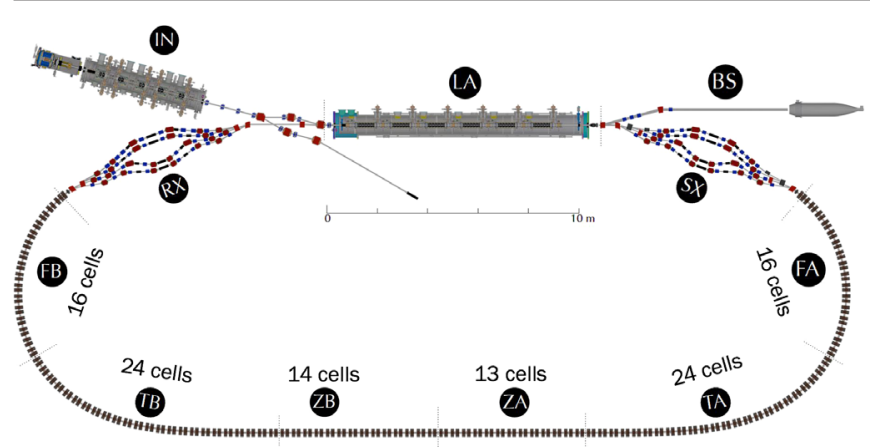

FIG. 1. Layout of CBETA. The sections labeled (IN) and (LA) are the injector and MLC cavities respectively. Sections (FA), (TA), (ZA), (ZB), (TB), and (FB) form the FFA beamline which can accommodate four recirculating orbits with energy ranging from $42 \mathrm{MeV}$ to $150 \mathrm{MeV}$. Sections (SX) and (RX) are splitters and recombiners which control the path-length of each recirculation pass.

While the beam current in ERLs is no longer limited by the power consumption in the cavities, there will be new, higher limits to the current. These are higher order modes (HOMs) heating and the recirculative beam breakup (BBU) instability. BBU occurs in recirculating accelerators as the recirculated beam bunches interact with the HOMs in the accelerating cavities. The most relevant HOMs for BBU are the dipole HOMs which give a transverse kick to the bunches. The off-orbit bunches return to the same cavity and excite the dipole HOMs which can kick the subsequent bunches further in the same direction. The effect can build up and can eventually result in beam loss. With a larger beam current the effect becomes stronger, so BBU is a limiting factor on the maximum achievable current, called the threshold current $I_{\text {th }}$. With multiple recirculation passes, bunches interact with cavities for multiple times, and the $I_{\text {th }}$ can significantly decrease [6]. The low and high target currents of CBETA are $1 \mathrm{~mA}$ and $40 \mathrm{~mA}$ respectively, for both the 1-pass mode and 4-pass mode. Simulations are required to check whether the $I_{\text {th }}$ is above these target values.

\section{BBU SIMULATION OVERVIEW}

Cornell University has developed a simulation software called BMAD to model relativistic beam dynamics in customized accelerator lattices [7]. Subroutines have been established to simulate specifically BBU and to find the $I_{\text {th }}$ for a specific lattice design. The program requires the lattice to have at least one recirculated cavity with at least one HOM assigned to it. There are six MLC cavities in the CBETA lattice, and multiple HOMs can be assigned to each cavity. The following two subsections describe how the HOM data are generated, and how BMAD finds the $I_{\text {th }}$.

\section{A. HOM simulation and assignment}

To run BBU simulation we must first obtain the HOM characteristics. Each HOM is characterized by its

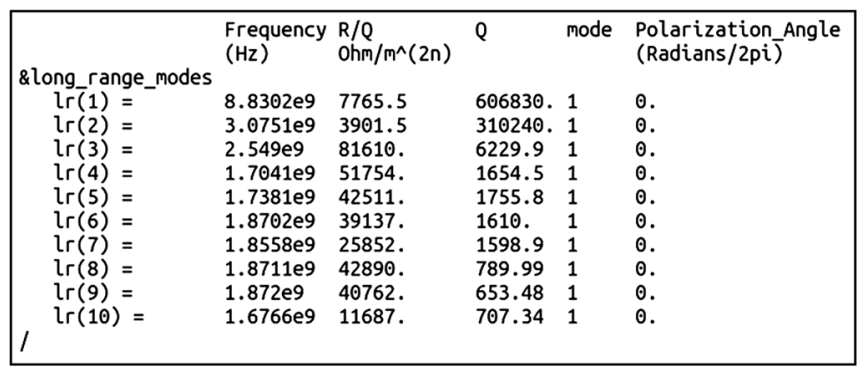

FIG. 2. An example file of 10 dominant horizontal dipoleHOMs assigned to a single CBETA MLC cavity. The HOMs are simulated using CLANS2 program with $\epsilon=125 \mu \mathrm{m}$. Note that all the HOM frequencies are above the fundamental frequency $1.3 \mathrm{GHz}$.

frequency $f$, shunt impedance $(R / Q)$, quality factor $Q$, order $m$, and polarization angle $\theta$. Since the MLC cavities have been built and commissioned, one would expect direct measurement of HOM spectra from the cavities. Unfortunately, the measured spectra contain hundreds of HOMs, and it is difficult to isolate each individual HOM and compute their characteristics, particularly $R / Q$. Therefore, instead of direct measurement, we simulate the HOM profiles using the known and modelled cavity structures [8]. The simulation has been done using the CLANS2 program [9], which can model the fields and HOM spectrum within a cavity.

In reality each cavity is manufactured with small unknown errors. The cavity shape are characterized by ellipse parameters. The fabrication tolerance for the CBETA MLC cavities require the errors in these parameters to be within $\pm 125 \mu \mathrm{m}$. For simplicity we use $\epsilon$ to denote the maximum deviation, i.e., $\epsilon=125 \mu \mathrm{m}$ for realistic CBETA cavities. In the CLANS2 program, random errors are introduced to the modeled cavity shape within a specified $\epsilon$. The cavity is then compressed to obtain the desired fundamental accelerating frequency. This procedure results in different HOM spectra for each cavity. Hundreds of spectra were generated, each representing a possible cavity in reality. The six MLC cavities in CBETA have different manufacturing errors, therefore each BBU simulation in BMAD assigns each cavity one of these precalculated HOM spectrum. With multiple BBU simulations we therefore obtain a statistical distribution of $I_{\text {th }}$ of CBETA because the assigned HOM spectra will be different for each BBU simulation.

To save simulation time we include only the 10 most dominant transverse dipole-HOMs $(m=1)$ from a precalculated spectrum. A dipole-HOM is considered more dominant if it has a greater figure-of-merit $\xi=(R / Q)$ $\sqrt{Q} / f$ [8]. Figure 2 shows an example HOM assignment file with 10 dipole-HOMs for $\epsilon=125 \mu \mathrm{m}$. The zero polarization angles indicate that all these HOMs are horizontally polarized which give no vertical kick to the beam bunches. We include only horizontal HOMs and exclude any vertical HOMs. This is a reasonable model 
since the cavities have cylindrical symmetry. For the rest of this paper, HOM refers to dipole-HOM unless further specified.

\section{B. BMAD simulation detail}

The goal of BBU simulations is to find the $I_{\text {th }}$ for a given multipass lattice with HOMs assigned to the cavities. The BMAD program starts with a test current by injecting beam bunches into the lattice at a constant repetition rate. The initial bunches populating the lattice are given small transverse orbit offsets to allow initial excitation of the HOMs. As the bunches pass through the cavities, the momentum exchange between the bunches and the wake fields are calculated, and the HOM voltages are updated. The program records all the HOM voltages over time and periodically examine their stability. If all $\mathrm{HOM}$ voltages are stable over time, the test current is considered stable, and a greater current will be tested. Since the repetition rate is held constant, this is equivalent to raising the charge per bunch. In contrast, if at least one HOM voltage is unstable, the test current is regarded unstable, and a smaller current will be tested. The program typically converges to a $I_{\text {th }}$ within $0.1 \%$ accuracy in under 30 iterations.

Since the BBU instability occurs because bunches interact with HOMs in the cavities, detailed tracking in the recirculation arc is not required. To save simulation time we usually hybridize the arc elements into an equivalent transfer matrix. The time advantage of hybridization is one to two orders of magnitude.

\section{BMAD SIMULATION RESULT}

As discussed, CBETA can operate in either the 1-pass or 4-pass mode, and each of the 6 MLC cavities can be assigned with a set of HOM spectrum. Hundreds of simulations with different HOM assignments were run to obtain a statistical distribution of $I_{\text {th }}$ for each specific CBETA design. We will investigate the following five design cases:

Case (1): CBETA 1-pass with $\epsilon=125 \mu \mathrm{m}$

Case (2): CBETA 4-pass with $\epsilon=125 \mu \mathrm{m}$

Case (3): CBETA 4-pass with $\epsilon=250 \mu \mathrm{m}$

Case (4): CBETA 4-pass with $\epsilon=500 \mu \mathrm{m}$

Case (5): CBETA 4-pass with $\epsilon=1000 \mu \mathrm{m}$

The first two cases aim to model the reality since CBETA cavities have the fabrication tolerance of $\pm 125 \mu \mathrm{m}$. The latter three cases with greater fabrication errors are simulated for academic interest. Results of all the cases are presented as histograms in the following subsections. Note that some of the results have been presented in [10].

\section{A. CBETA 1-pass with $\boldsymbol{\epsilon}=\mathbf{1 2 5} \boldsymbol{\mu \mathrm { m }}$}

The design current of CBETA 1-pass mode is $1 \mathrm{~mA}$ (the low goal) and $40 \mathrm{~mA}$ (the high goal). It is important to note that these goals refer to the injected current, so a $40 \mathrm{~mA}$

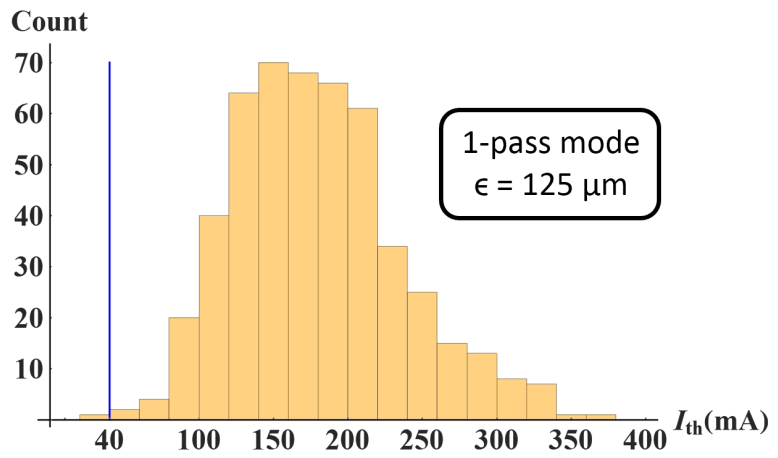

FIG. 3. 500 BBU simulation results of $I_{\text {th }}$ for the CBETA 1-pass lattice. Each cavity is assigned with a random set of 10 dipole HOMs $(\epsilon=125 \mu \mathrm{m})$. The blue line indicates the higher current goal of $40 \mathrm{~mA}$.

injected current corresponds to $80 \mathrm{~mA}$ for the 1-pass mode $\left(N_{p}=2\right)$ and $320 \mathrm{~mA}$ for the 4-pass mode $\left(N_{p}=8\right)$ at the MLC cavities. Figure 3 shows that all 500 simulations results exceed the lower goal of $1 \mathrm{~mA}$, and only one of them is below $40 \mathrm{~mA}$. This is a promising result for the CBETA 1-pass operation. We have to be unfortunate for the cavities to assume certain undesirable combinations of HOMs for the current to not reach the high goal.

\section{B. CBETA 4-pass with $\boldsymbol{\epsilon}=\mathbf{1 2 5} \boldsymbol{\mu \mathrm { m }}$}

The design current of CBETA 4-pass mode is also $1 \mathrm{~mA}$ and $40 \mathrm{~mA}$. Figure 4 shows that for the 4-pass mode, 494 out of 500 simulations exceed the $40 \mathrm{~mA}$ goal. This is again quite promising for the 4-pass operation, and for the few cases with undesirably low $I_{\text {th }}$, we will discuss the potential ways to improve them in the following section. Comparing to Fig. 3, the average $I_{\text {th }}$ for the 4-pass mode is $80.8 \mathrm{~mA}$, much lower than the $179.4 \mathrm{~mA}$ of the 1-pass mode. This is expected from the BBU theory, since more recirculation passes allow more interaction between the HOMs and beam bunches, thus resulting in a smaller $I_{\text {th }}$.

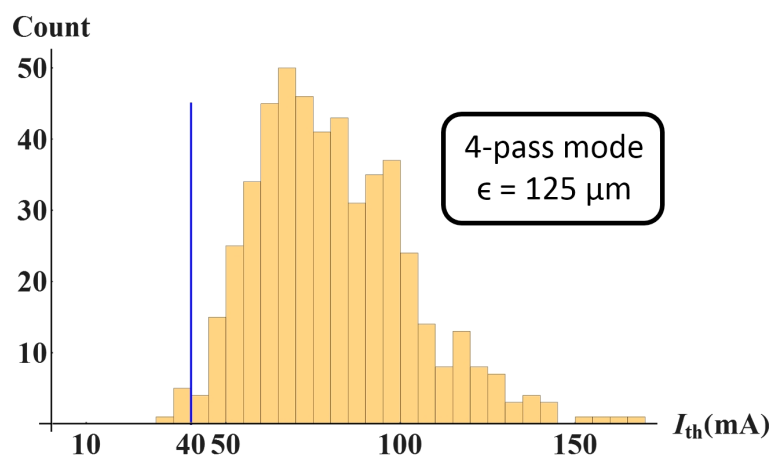

FIG. 4. $500 \mathrm{BBU}$ simulation results of $I_{\text {th }}$ for the CBETA 4-pass lattice. Each cavity is assigned with a random set of 10 dipole HOMs $(\epsilon=125 \mu \mathrm{m})$. 


\section{CBETA 4-pass with $\epsilon \geq 250 \mu \mathrm{m}$}

It is interesting to see how $I_{\text {th }}$ distribution changes with greater manufacture errors for the 4-pass lattice. Figures 5, 6, and 7 show the results of 500 simulations for $\epsilon=250 \mu \mathrm{m}, \epsilon=500 \mu \mathrm{m}$, and $\epsilon=1000 \mu \mathrm{m}$ respectively. For simple comparison, Table I summarizes the statistics of all the results. For $\epsilon=250 \mu \mathrm{m}$, the minimum and average $I_{\text {th }}$ are both higher than the $\epsilon=125 \mu \mathrm{m}$ case.

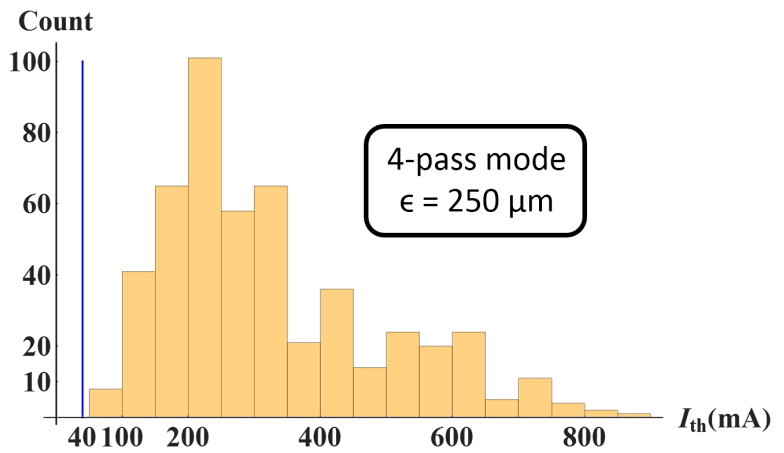

FIG. 5. $500 \mathrm{BBU}$ simulation results of $I_{\mathrm{th}}$ for the 4-pass lattice with cavity shape errors within $\epsilon=250 \mu \mathrm{m}$.

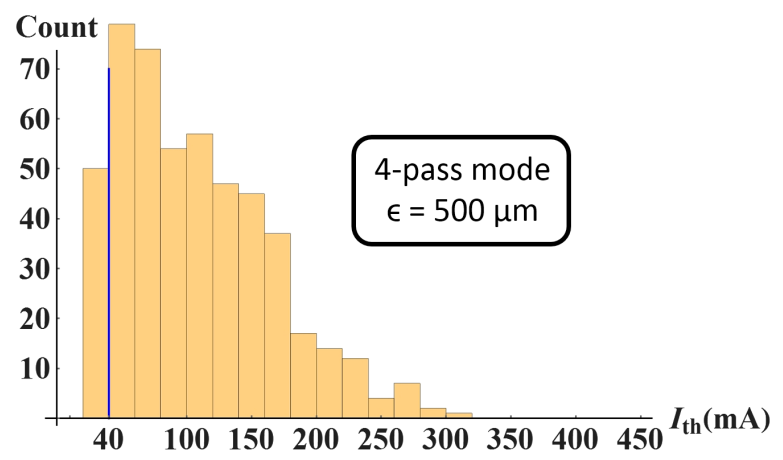

FIG. 6. $500 \mathrm{BBU}$ simulation results of $I_{\mathrm{th}}$ for the 4-pass lattice with cavity shape errors within $\epsilon=500 \mu \mathrm{m}$.

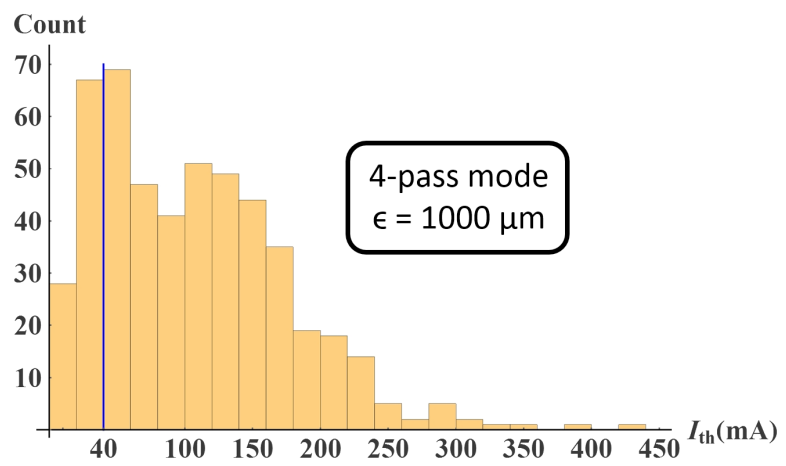

FIG. 7. $500 \mathrm{BBU}$ simulation results of $I_{\mathrm{th}}$ for the 4-pass lattice with cavity shape errors within $\epsilon=1000 \mu \mathrm{m}$.
TABLE I. Summary of the BBU $I_{\text {th }}$ statistics of different CBETA design cases. For the 4-pass mode, $\epsilon=250 \mu \mathrm{m}$ generates the most satisfying $I_{\text {th }}$ statistics.

\begin{tabular}{lccccc}
\hline \hline $\begin{array}{l}\text { CBETA } \\
\text { mode } \\
\left(N_{p} / 2\right)\end{array}$ & $\begin{array}{c}\epsilon \\
(\mu \mathrm{m})\end{array}$ & $\begin{array}{c}\mu\left(I_{\mathrm{th}}\right) \\
(\mathrm{mA})\end{array}$ & $\begin{array}{c}\sigma\left(I_{\text {th }}\right) \\
(\mathrm{mA})\end{array}$ & $\begin{array}{c}\min \left(I_{\text {th }}\right) \\
(\mathrm{mA})\end{array}$ & $\begin{array}{c}\mathrm{N} \text { in } 500 \\
\text { cases with } \\
I_{\text {th }}<40 \mathrm{~mA}\end{array}$ \\
\hline 1-pass & 125 & 179.4 & 56.1 & 21.9 & 1 \\
4-pass & 125 & 80.8 & 22.4 & 34.4 & 6 \\
4-pass & 250 & 325.3 & 164.4 & 82.4 & 0 \\
4-pass & 500 & 107.1 & 59.1 & 20.4 & 50 \\
4-pass & 1000 & 106.6 & 69.3 & 8.8 & 95 \\
\hline \hline
\end{tabular}

However, the low average $I_{\text {th }}$ for $\epsilon=1000 \mu \mathrm{m}$ implies that a greater $\epsilon$ does not always improve the $I_{\text {th }}$.

Fundamentally greater deviation in the cavity shape results in greater spread in the HOM frequencies. This causes the HOMs across the cavities to act less coherently when kicking the beam, thus potentially increases the $I_{\text {th }}$. However, a greater deviation also tends to undesirably increase the $Q$ and $R / Q$ of the HOMs, which usually lowers the $I_{\text {th }}$. This could explain why $I_{\text {th }}$ statistics improves as $\epsilon$ increases from $125 \mu \mathrm{m}$ to $250 \mu \mathrm{m}$, but deteriorates at $1000 \mu \mathrm{m}$. Compensation between the frequency spread and HOM damping also implies that an optimal manufacture tolerance could exist to raise the overall $I_{\text {th }}$.

\section{AIM FOR HIGHER $\boldsymbol{I}_{\text {th }}$}

From BBU theory we know that $I_{\text {th }}$ depends generally on the HOM properties $\left(\omega_{\lambda}, Q_{\lambda},(R / Q)_{\lambda}\right)$, the lattice properties $\left(t_{r}\right.$ and $\left.T_{12}\right)$, and the injected bunch time spacing $t_{b}$. The previous section shows how $I_{\text {th }}$ can vary with different HOM spectra in the cavities. Our goal now is to study how much the $I_{\text {th }}$ of CBETA can be improved. Based on the knowledge from BBU theory, three methods have been proposed and tested for existing 1-turn ERLs:

Method (1) Vary $t_{b}$

Method (2) Vary phase advance

Method (3) Introduce x-y coupling

Both the second and third method involve modifying the optics of the recirculation beamline between the pairs of multipass cavities. The idea of modifying beam optics to improve the $I_{\text {th }}$ was first suggested in 1980 [11], and has been tested out at the Jefferson Lab's free electron laser [12-14]. The effect of all three methods are simulated using BMAD following only the design particle, with results presented in the three following subsections.

When phase space filamentation is relevant, the BBU instability can be suppressed, e.g., when the chromaticity times the energy spread is of order 1 [15]. Since the $42 \mathrm{MeV}$ orbit in the FFA lattice has a great natural chromaticity, additional simulations are performed for the CBETA 1-pass lattice. Note that in order to demonstrate the chromaticity effect, it requires tracking of multiple particles per bunch with an energy spread. This new feature 


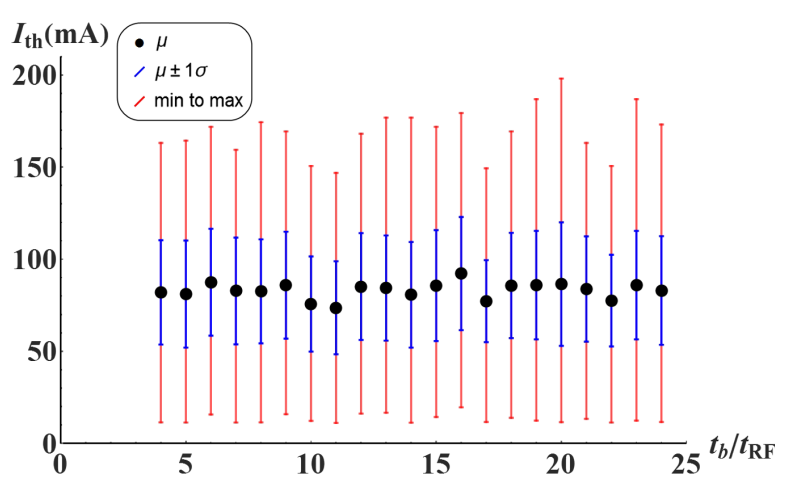

FIG. 8. $I_{\text {th }}$ vs. $t_{b} / t_{\mathrm{rf}}$ for the CBETA 4-pass lattice. For each $t_{b} / t_{\mathrm{rf}}, 500$ simulations are run with different HOM assignments $(\epsilon=125 \mu \mathrm{m})$. The black dot marks the average $I_{\text {th }}$, and the blue inner line marks the $\pm 1 \sigma$ range. The red outer line marks the range of the entire distribution.

has been implemented in BBU simulation of BMAD. The results and related theoretical analysis are presented in Sec. VII.

\section{A. Effect on $\boldsymbol{I}_{\text {th }}$ by varying $\boldsymbol{t}_{\boldsymbol{b}}$}

Equation (8) and Eq. (10) show that the $I_{\text {th }}$ depends on $t_{b}$ in a complicated way even for the most elementary BBU case. The dependence however vanishes in the approximate formula for the trough region [Eq. (13)]. It is interesting to investigate how $I_{\text {th }}$ of CBETA varies with $t_{b}$ using simulation. For all the bunches to see desired longitudinal acceleration, $t_{b}=n t_{\mathrm{rf}}$ is required with a positive integer $n$.

For the rest of the paper, let $N_{p}$ denote the number of times a bunch traverses the multipass cavity(s), which is always equal to the number of recirculations plus one. For an ERL, $N_{p}$ must be an even number, since each pass through a cavity for acceleration is accompanied by one for deceleration. For instance, the CBETA 1-pass lattice has $N_{p}=2$ and one recirculation, while the 4-pass lattice has $N_{p}=8$ and seven recirculations. Traditionally such an accelerator is referred to as an $N_{p} / 2$ turn ERL.

For all the CBETA results presented in the previous section, we have set $n=N_{p} / 2$. This corresponds to filling all the rf buckets (i.e., CW operation), and practically we would not use a smaller $n$ to avoid overlapping bunches. Figure 8 shows the simulated $I_{\text {th }}$ statistics with increasing $n$ at integer steps for the 4-pass lattice $\left(N_{p}=8, \min [n]=4\right)$. To focus on the effect of varying $t_{b}$ only, the 500 sets of HOM assignments are fixed. The result shows that the $I_{\text {th }}$ depends weakly on $t_{b}$, and potential improvement on $I_{\text {th }}$ is limited. Specifically the average $I_{\text {th }}$ does not change by $5 \%$. It will still be interesting to test the effect of varying $t_{b}$ when CBETA begins operation.

\section{B. Effect on $\boldsymbol{I}_{\text {th }}$ by varying phase advance}

$I_{\text {th }}$ can potentially be improved by changing the phase advances (in both $\mathrm{x}$ and $\mathrm{y}$ ) between the multipass cavities.
This method equivalently changes the $T_{12}$ (and $T_{34}$ ) element of the transfer matrices. In the elementary case of BBU theory, smaller $T_{12}$ directly results in greater $I_{\text {th }}$ [Eq. (8)]. However, with multiple cavities and HOMs, it is generally difficult to lower all the $T_{12}$ elements between different HOM pairs. To freely vary the phase advances in BMAD simulations, a zero-length lattice element is introduced right after the first pass of the MLC cavities. The element has the following $4 \times 4$ transfer matrix in the transverse phase space:

$$
T_{\text {decoupled }}\left(\phi_{x}, \phi_{y}\right)=\left(\begin{array}{cc}
M_{x \leftarrow x}\left(\phi_{x}\right) & \mathbf{0} \\
\mathbf{0} & M_{y \leftarrow y}\left(\phi_{y}\right)
\end{array}\right) .
$$

Each of the $2 \times 2$ submatrix depends on the Twiss parameters $\left(\beta_{i}, \alpha_{i}\right.$, and $\left.\gamma_{i}\right)$ in one transverse direction $(i=x$ or $y)$ at the location of introduction:

$M_{i \leftarrow i}(\phi)=\left(\begin{array}{cc}\cos \phi+\alpha_{i} \sin \phi & \beta_{i} \sin \phi \\ -\gamma_{i} \sin \phi & \cos \phi-\alpha_{i} \sin \phi\end{array}\right)$.

Note that $\phi_{x}$ and $\phi_{y}$ are the additional transverse phase advances introduced by the element, and both can be chosen freely between $[0,2 \pi)$. The $4 \times 4$ matrix does not introduce optical coupling between the two transverse phase spaces, and is thus named $T_{\text {decoupled. }}$. In reality there is no physical element providing such a flexible transfer matrix, and the phase advances are changed by adjusting the quad strengths around the accelerator structure. In simulation the introduction of this matrix allows us to arbitrarily yet effectively vary the two phase advances.

To investigate how $I_{\text {th }}$ varies with both transverse lattice optics, we need to include vertical HOMs which give vertical kicks to the bunches. Therefore for each simulation, each cavity is assigned with three dominant " $e=$ $125 \mu \mathrm{m}$ " horizontal HOMs and three identical vertical HOMs (polarization angle $=\pi / 2$ ). Figure 9 shows an example assignment to one cavity. With a fixed set of HOM assignments, the $I_{\text {th }}$ statistics is obtained for different choices of $\left(\phi_{x}, \phi_{y}\right)$.

One hundred statistics were obtained for both the 1-pass and 4-pass CBETA lattice, and typical statistics are shown by Figs. 10 and 11 respectively. Depending on the HOM

\begin{tabular}{|clllll|}
\hline \multicolumn{7}{c}{$\begin{array}{l}\text { Frequency } \\
(\mathrm{Hz})\end{array}$} & $\begin{array}{l}\mathrm{R} / \mathrm{Q} \\
\mathrm{Ohm} / \mathrm{m}^{\wedge}(2 \mathrm{n})\end{array}$ & $\mathrm{Q}$ & Mode & $\begin{array}{l}\text { Polarization_Angle } \\
(\text { Radians/2pi })\end{array}$ \\
\&long_range_modes & & & & \\
$\operatorname{lr}(1)=$ & $2.5512 \mathrm{e} 9$ & 79593.0 & 6100.8 & 1 & 0.0 \\
$\operatorname{lr}(2)=$ & $8.8345 \mathrm{e} 9$ & 8005.2 & 439340.0 & 1 & 0.0 \\
$\operatorname{lr}(3)=$ & $8.8345 \mathrm{e} 9$ & 7907.8 & 350650.0 & 1 & 0.0 \\
$\operatorname{lr}(4)=$ & $2.5512 \mathrm{e} 9$ & 79593.0 & 6100.8 & 1 & 0.25 \\
$\operatorname{lr}(5)=$ & $8.8345 \mathrm{e} 9$ & 8005.2 & 439340.0 & 1 & 0.25 \\
$\operatorname{lr}(6)=$ & $8.8345 \mathrm{e} 9$ & 7907.8 & 350650.0 & 1 & 0.25 \\
$\ln$ & & & & & \\
\hline
\end{tabular}

FIG. 9. An example file of three dominant horizontal HOMs (the top 3) and three identical vertical HOMs (the bottom 3) assigned to a single CBETA MLC cavity. The HOMs are simulated using HTC program with $\epsilon=125 \mu \mathrm{m}$. 


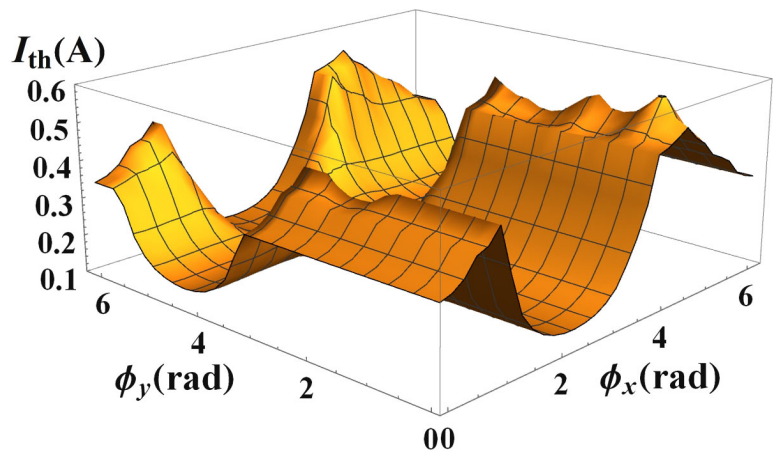

FIG. 10. A scan of BBU $I_{\text {th }}$ over the two phase advances for the CBETA 1-pass lattice. Each cavity is assigned with a random set of 3 dipole HOMs in both $\mathrm{x}$ and y polarization. ( $\epsilon=125 \mu \mathrm{m})$. For this particular HOM assignment, $I_{\text {th }}$ ranges from $140 \mathrm{~mA}$ to $610 \mathrm{~mA}$.

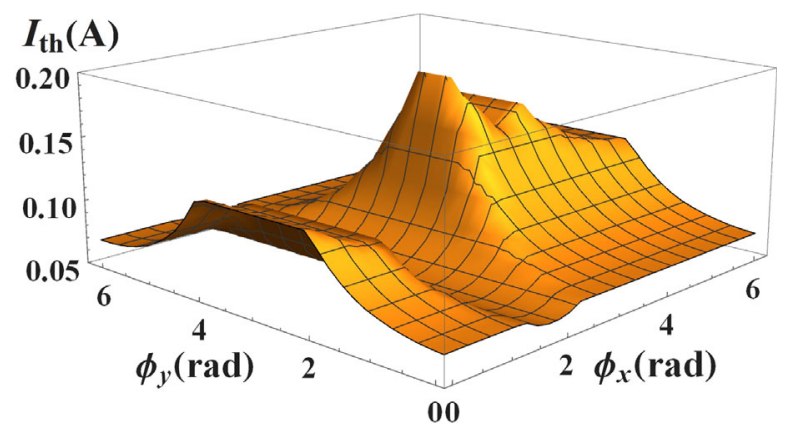

FIG. 11. A scan of BBU $I_{\text {th }}$ over the two phase advances for the CBETA 4-pass lattice. Each cavity is assigned with a random set of 3 dipole HOMs in both $\mathrm{x}$ and y polarization. ( $\epsilon=125 \mu \mathrm{m})$. For this particular HOM assignment, $I_{\text {th }}$ ranges from $61 \mathrm{~mA}$ to $193 \mathrm{~mA}$.

assignment, the peak $I_{\text {th }}$ can reach at least $461 \mathrm{~mA}$ for the 1-pass mode (and $171 \mathrm{~mA}$ for the 4-pass mode) with an optimal choice of $\left(\phi_{x}, \phi_{y}\right)$. Table II summarizes the statistics of the peak $I_{\text {th }}$ with the 100 different HOM assignments. Clearly varying phase advances can be used to (significantly) improve the $I_{\text {th }}$.

It is also observed that $\phi_{x}$ and $\phi_{y}$ affect $I_{\text {th }}$ rather independently. That is, at certain $\phi_{x}$ which results in a

TABLE II. Summary of the peak $I_{\text {th }}$ statistics with varying transverse optics over 100 different HOM-assignments for the CBETA 1-pass and 4-pass mode. For both modes, introducing additional phase advances (decoupled optics) gives greater potential to increase $I_{\text {th }}$ than X-y coupling.

\begin{tabular}{lccc}
\hline \hline Case (optics) & $\begin{array}{c}\min \left(\text { peak } I_{\text {th }}\right) \\
(\mathrm{mA})\end{array}$ & $\begin{array}{c}\mu\left(\text { peak } I_{\text {th }}\right) \\
(\mathrm{mA})\end{array}$ & $\begin{array}{c}\max \left(\text { peak } I_{\text {th }}\right) \\
(\mathrm{mA})\end{array}$ \\
\hline 1-pass (decoupled) & 461 & 733 & 1275 \\
1-pass (coupled) & 299 & 557 & 928 \\
4-pass (decoupled) & 171 & 440 & 758 \\
4-pass (coupled) & 127 & 434 & 548 \\
\hline \hline
\end{tabular}

low $I_{\text {th }}$ (the "valley"), different choice of $\phi_{y}$ does not help increase $I_{\text {th }}$, and vice versa. It is also observed that $I_{\text {th }}$ is more sensitive to $\phi_{x}$, and the effect of $\phi_{y}$ becomes obvious mostly at the "peak" in $\phi_{x}$. Physically this is expected since many lattice elements have a unit transfer matrix in the vertical phase space, and the effect of varying $T_{12}$ is more significant than $T_{34}$. In other words, HOMs with horizontal polarization are more often excited. As we will see this is no longer true when $x-y$ coupling is introduced.

It is also observed that the location of the valley remains almost fixed when HOM assignments are similar. Physically the valley occurs when the combination of phase-advances results in a great equivalent $T_{12}$ (or $T_{34}$ ) which excites the most dominant HOM. Therefore, the valley location depends heavily on which cavity has the most dominant HOM, and the simulation results agree with this observation.

In reality the optimal set of $\left(\phi_{x}, \phi_{y}\right)$ to achieve the peak $I_{\text {th }}$ may not be achievable for CBETA due to its limited number of free quadrupole magnets and strict constraints on beam optics. However it suffices to have enough freedom to increase the $I_{\text {th }}$ over the design goal of $40 \mathrm{~mA}$ for the 4-pass mode. It will be interesting to investigate this experimentally at CBETA.

\section{Effect on $\boldsymbol{I}_{\text {th }}$ with $x-y$ coupling}

Another method potentially improves $I_{\text {th }}$ by introducing $\mathrm{x}-\mathrm{y}$ coupling in the transverse optics, so that horizontal HOMs excite vertical motions and vise versa. This method has been shown very effective for 1-pass ERLs [16]. To simulate the coupling effect in BMAD simulation, a different $4 \times 4$ matrix of zero-length is again introduced right after the first pass of the LINAC:

$$
T_{\text {coupled }}\left(\phi_{1}, \phi_{2}\right)=\left(\begin{array}{cc}
\mathbf{0} & M_{x \leftarrow y}\left(\phi_{1}\right) \\
M_{y \leftarrow x}\left(\phi_{2}\right) & \mathbf{0}
\end{array}\right) .
$$

The elements of the two $2 \times 2$ submatrices $M_{j \leftarrow i}(\phi)$ are specified using on the transverse Twiss parameters at the location of introduction:

$$
\begin{aligned}
& M_{11}=\sqrt{\frac{\beta_{j}}{\beta_{i}}}\left(\cos \phi+\alpha_{i} \sin \phi\right) \\
& M_{12}=\sqrt{\beta_{j} \beta_{i}} \sin \phi \\
& M_{21}=\frac{1}{\sqrt{\beta_{j} \beta_{i}}}\left[\left(\alpha_{i}-\alpha_{j}\right) \cos \phi-\left(1+\alpha_{i} \alpha_{j}\right) \sin \phi\right] \\
& M_{22}=\sqrt{\frac{\beta_{j}}{\beta_{i}}}\left(\cos \phi-\alpha_{j} \sin \phi\right) .
\end{aligned}
$$

The symplectic $4 \times 4$ matrix $T_{\text {coupled }}$ couples the lattice optics in the two transverse directions with two phases of 


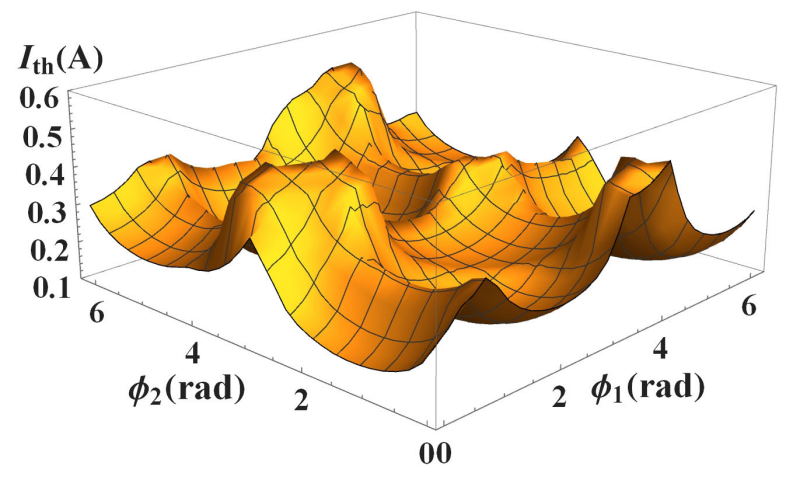

FIG. 12. A scan of BBU $I_{\text {th }}$ over the two free phases for the CBETA 1-pass lattice with $x-y$ coupling. Each cavity is assigned with a random set of 3 dipole HOMs in both $\mathrm{x}$ and $\mathrm{y}$ polarization. $(\epsilon=125 \mu \mathrm{m})$. For this particular HOM assignment, $I_{\text {th }}$ ranges from $140 \mathrm{~mA}$ to $520 \mathrm{~mA}$.

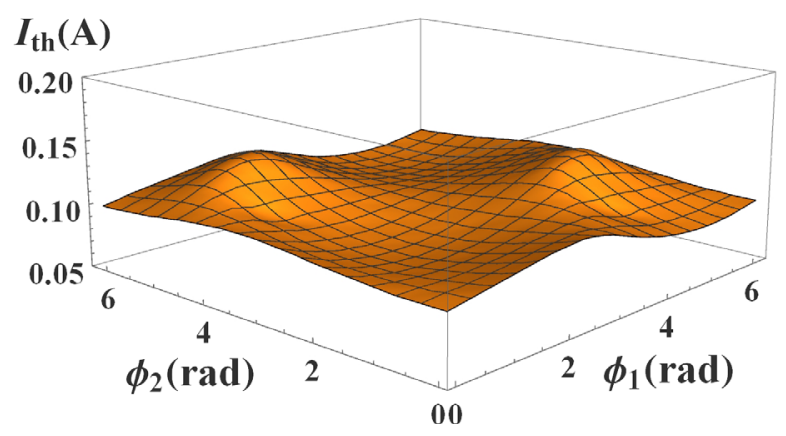

FIG. 13. A scan of BBU $I_{\text {th }}$ over the two free phases for the CBETA 4-pass lattice with $x-y$ coupling. Each cavity is assigned with a random set of 3 dipole HOMs in both $\mathrm{x}$ and y polarization. $(\epsilon=125 \mu \mathrm{m})$. For this particular HOM assignment, $I_{\text {th }}$ ranges from $89 \mathrm{~mA}$ to $131 \mathrm{~mA}$.

free choice $\left(\phi_{1}, \phi_{2}\right)$. Note the two phases are not the conventional phase advances, and can both range from 0 to $2 \pi$.

Figures 12 and 13 show a typical way $I_{\text {th }}$ varies with the two free phases for the 1-pass and 4-pass lattice respectively. Depending on the HOM assignment, the $I_{\text {th }}$ can reach at least $299 \mathrm{~mA}$ for the 1-pass mode (and $127 \mathrm{~mA}$ for the 4-pass mode) with an optimal choice of $\left(\phi_{1}, \phi_{2}\right)$. Because the transverse optics are coupled, the two phases no longer affect $I_{\text {th }}$ in an independent manner. That is, there is no specific $\phi_{1}$ which would always result in a relatively high or low $I_{\text {th }}$. The two phases need to be varied together to reach the peak $I_{\text {th }}$.

Similar to the case with decoupled optics, 100 statistics are run for both the 1-pass and 4-pass mode with different HOM assignments, and the statistics of the peak $I_{\text {th }}$ are summarized in Table II. As expected from theory, the $I_{\text {th }}$ can statistically reach a higher value for the 1-pass mode than the 4-pass mode. While introducing additional phase advances and $x-y$ coupling both give great potential to raise the peak $I_{\text {th }}$ (way above the high design goal of $40 \mathrm{~mA}$ ), the former gives more. In realty, introducing $x-y$ coupling also requires installation of skew quadrupole magnets, and CBETA might not achieve this due to limited space. In short, varying phase advances is the most promising method to improve the $I_{\text {th }}$ of CBETA.

\section{BBU THEORY VS. BMAD SIMULATION}

In this section we will revisit the general BBU theory developed in [6] and [16] to check the validity of BMAD simulations by comparing the results to the theory predictions. Benchmarking with numerical code has been performed in [6] for the most elementary BBU configuration (one dipole-HOM with one recirculation) using the recirculation arc time $t_{r}$ as the variable. However, CBETA is a multipass ERL with multiple cavities. To ensure Bmad simulation results are representative, we also benchmark the code with more complicated configurations with various $t_{r}$. This has not been done in the previously publications. Furthermore, by using similar lattice and HOM parameters across the configurations, one could compare the effect on the $I_{\text {th }}$ in a consistent fashion. We believe these benchmarking results will be important to CBETA as well as future multipass ERLs which concern BBU.

We will focus on four configurations (cases) of which analytic formulas for $I_{\text {th }}$ are available from $[6,16]$ :

Case A: One dipole-HOM with $N_{p}=2$,

Case B: One dipole-HOM with $N_{p}=4$,

Case C: One dipole-HOM in two different cavities with $N_{p}=2$,

Case D: Two polarized dipole-HOMs in one cavity with $N_{p}=2$.

Since the theory assumes thin-lens cavities, it is inaccurate to benchmark with the CBETA lattice whose cavities are each $1 \mathrm{~m}$ long. Instead we make a simple lattice with only thin-lens cavities and a recirculation arc with fixed optics. The following subsections compare the simulation results to theoretical formulas for the four cases.

\section{A. One dipole-HOM with $N_{p}=\mathbf{2}$}

Case A is the most elementary case for BBU. Assuming that the injected current $I_{0}$ consists of a continuous stream of bunches with a constant charge and separated by a constant time interval $t_{b}$, then the time-dependent HOM voltage $V(t)$ must satisfy, for any positive integer $n$, the recursive equation [6]:

$V\left(n t_{b}+t_{r}\right)=I_{0} \frac{e}{c} t_{b} T_{12} \sum_{m=0}^{\infty} W\left(m t_{b}\right) V\left([n-m] t_{b}\right)$,

in which $W(\tau)$ is the long range wake function characterized by the HOM parameters:

$$
W(\tau)=\left(\frac{R}{Q}\right)_{\lambda} \frac{\omega_{\lambda}^{2}}{2 c} e^{-\left(\omega_{\lambda} / 2 Q_{\lambda}\right) \tau} \sin \left(\omega_{\lambda} \tau\right) .
$$


TABLE III. A list of important quantities in the elementary BBU theory (one dipole-HOM, $N_{p}=2$ ). $\epsilon$ is a measure of HOM decay in the time scale of $t_{b}$.

\begin{tabular}{|c|c|c|}
\hline Symbol & SI Unit & Definition or Meaning \\
\hline$e$ & $\mathrm{C}$ & Elementary charge \\
\hline$c$ & $\mathrm{~m} / \mathrm{s}$ & Speed of light \\
\hline$t_{\mathrm{rf}}$ & s & Fundamental rf period \\
\hline$t_{b}$ & $\mathrm{~s}$ & Injected bunch time spacing $\left(>t_{\mathrm{rf}}\right)$ \\
\hline$t_{r}$ & $\mathrm{~s}$ & Recirculation arc time (typically $>t_{b}$ ) \\
\hline$n_{r}$ & $\cdots$ & $n_{r}=\operatorname{Top}\left[t_{r} / t_{b}\right]$, integer \\
\hline$\delta$ & $\cdots$ & $\begin{array}{l}\delta=\left(t_{r} / t_{b}-n_{r}\right) \in[0,1) \\
\text { For an ERL } \delta \approx 0.5\end{array}$ \\
\hline$\omega_{\lambda}$ & $\mathrm{rad} / \mathrm{s}$ & HOM radial frequency \\
\hline$(R / Q)_{\lambda}$ & $\Omega$ & normalized HOM Shunt Impedance \\
\hline$Q_{\lambda}$ & $\cdots$ & HOM quality factor \\
\hline$T_{12}$ & $\mathrm{~s} / \mathrm{kg}$ & $\begin{array}{l}\text { The } T_{12} \text { element of the transfer } \\
\text { matrix of the recirculation arc }\end{array}$ \\
\hline$W(\tau)$ & $\mathrm{V} / \mathrm{mC}$ & Long range wake function [see Eq. (6)] \\
\hline$w(\delta, \omega)$ & $\mathrm{V} / \mathrm{mC}$ & Sum over all wakes [see Eq. (10)] \\
\hline$I_{0}$ & A & Measured current at the injector \\
\hline$\epsilon$ & $\cdots$ & $\epsilon=\left(\omega_{\lambda} / 2 Q_{\lambda}\right) t_{b}$ \\
\hline$\kappa$ & $\mathrm{Cs} \Omega / \mathrm{m}^{2}$ & $\kappa=t_{b}\left(e / c^{2}\right)(R / Q)_{\lambda}\left(\omega_{\lambda}^{2} / 2\right)$ \\
\hline
\end{tabular}

All the related symbols are listed in Table III, which closely follows the nomenclature used in [6].

The bunches arrive in the cavity at times $n t_{b}$, where they receive a transverse kick proportional to $V\left(n t_{b}\right)$, which then describes the transverse offset of successive bunches in the return loop. The Fourier transform

$$
\tilde{V}^{\Sigma}(\omega)=t_{b} \sum_{n=-\infty}^{\infty} V\left(n t_{b}\right) e^{i \omega n t_{b}}
$$

is zero for every $\omega$ except when the following dispersion relation is satisfied:

$$
\begin{gathered}
\frac{1}{I_{0}}=D(\omega), \\
D(\omega)=\frac{e}{c} t_{b} T_{12} e^{i \omega n_{r} t_{b}} w(\delta, \omega) .
\end{gathered}
$$

The function $w(\delta, \omega)$ sums the contribution of all the long range wakes in the frequency domain:

$$
w(\delta, \omega) \equiv \sum_{n=0}^{\infty} W\left([n+\delta] t_{b}\right) e^{i \omega n t_{b}} .
$$

As a current, $I_{0}$ is a real number, and for a fixed $I_{0}$ there is a set of complex values of $\omega$ which satisfy Eq. (8). For a small $I_{0}$ the voltage is stable, which means all the $\omega$ values have a negative imaginary part. If we keep increasing $I_{0}$, eventually instability will occur due to great excitement. This is reflected by the $\omega \mathrm{s}$ that have positive imaginary parts. At the onset of instability, one of the $\omega$ is crossing the real axis (i.e., is real), and the corresponding current $I_{0}$ is then the threshold current $I_{\text {th }}$. While it's difficult to find the

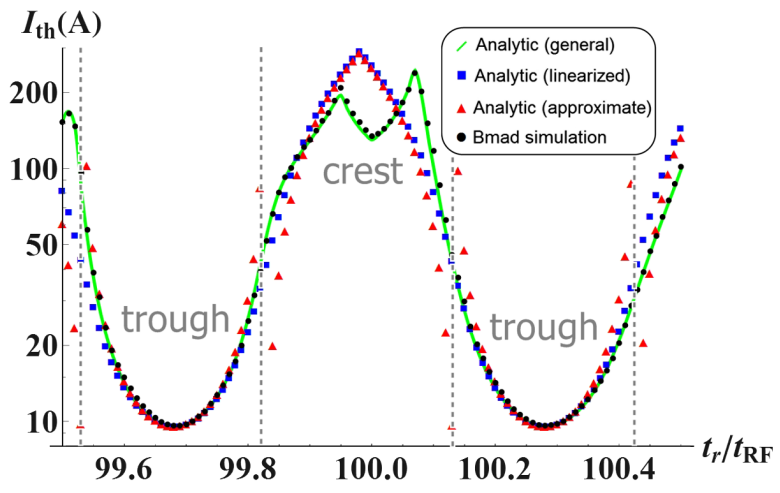

FIG. 14. Comparison of the $I_{\text {th }}$ obtained from different analytic formulas and BMAD simulation for $N_{p}=2$. Parameters: $c t_{\mathrm{rf}}=$ $0.5 \mathrm{~m}, t_{b}=50 t_{\mathrm{rf}}, \omega_{\lambda} / 2 \pi=1 \mathrm{GHz}, Q_{\lambda}=100,(R / Q)_{\lambda}=10^{4}$ $\Omega, T_{12}=-10 \mathrm{~m} /(1 \mathrm{GeV} / c)$.

$\omega$ values for a given $I_{0}$, it is easy to compute $D(\omega)$ given a real $\omega$. Most values computed will be complex and therefore correspond to an unphysical $I_{0}$. The largest real value of $D(\omega)$ determines the $I_{\mathrm{th}}$. Due to the periodicity and symmetry in Eq. (8), it is sufficient to check $\omega$ in just $\left[0, \pi / t_{b}\right)$ or any equivalent interval. Mathematically this can be written as:

$$
\frac{1}{I_{\text {th }}}=\max _{\omega}\left[D(\omega), D(\omega) \in \Re, \omega \in\left[0, \pi / t_{b}\right)\right] .
$$

Equation (8), combined with Eq. (11), is called the "general analytic formula" to determine the $I_{\text {th }}$ for case A. For a representative comparison between theory and simulation, we check how $I_{\text {th }}$ varies with $t_{r}$ while holding $t_{b}$ constant. The matrix element $T_{12}$ and the HOM properties are also held constant. Figure 14 shows the comparison result. Clearly BMAD's simulation agrees well with the general analytic formula, in both the regions with a high $I_{\text {th }}$ (the crest) and low $I_{\text {th }}$ (the trough).

If the HOM decay is insignificant on the timescale of $t_{b}$ $(\epsilon \ll 1)$, then Eq. $(8)$ can be simplified by linearization in small $\epsilon$. We call the resulting formula the "linearized analytic formula":

$$
D(\omega)=-\frac{\kappa}{2} \frac{e^{i \omega t_{r}} T_{12}}{\left(\omega-\omega_{\lambda}\right) t_{b}+i \epsilon} .
$$

Similar to the general formula, the linearized formula does not provide a closed form for $I_{\text {th }}$, so we still need to apply Eq. (11) to find the $I_{\text {th }}$ as the smallest real $I_{0}$ over $\omega \in\left[0, \pi / t_{b}\right)$.

The usefulness of the linearized formula will be shown when $N_{p}>2$. On the other hand, if the HOM decay is insignificant also on the recirculation time scale $\left(n_{r} \epsilon \ll 1\right)$, then the formula can be further simplified into the "approximate analytic formula": 


$$
I_{\mathrm{th}}= \begin{cases}-\frac{\epsilon}{\kappa} \frac{2}{T_{12} \sin \left(\omega_{\lambda} t_{r}\right)} & \text { if } T_{12} \sin \left(\omega_{\lambda} t_{r}\right)<0 \\ \frac{2}{\kappa\left|T_{12}\right|} \sqrt{\epsilon^{2}+\left(\frac{t_{b}}{t_{r}}\right)^{2} \times \operatorname{minmod}\left(\omega_{\lambda} t_{r}, \pi\right)} & \text { otherwise, }\end{cases}
$$

in which

$\min \bmod (x, y)=\min [\bmod (x, y), y-\bmod (x, y)]$.

It is worth checking the applicability of the linearized and the approximate formula. This has been done in [6] for a case with $\epsilon=0.00048$ and $n_{r}=6$ to 7 . Their result shows great agreement with the two nongeneral formulas in the trough region, but not in the crest region. Here we test a new case with $\epsilon=0.024$ and $n_{r}=2$ to 3 , and the results are plotted together on Fig. 14.

We again observe that the linearized formula agrees well with the general formula in the trough region, but the approximate formula agrees well only in a smaller region around the minimum of the trough. The inaccuracy of the approximate formula in the trough region comes from the increased value of $n_{r} \epsilon$. With HOM dampers, $Q_{\lambda}$ is typically on the order of $10^{4}$, so for an ERL with continuous wave operation $\left(t_{b}=2 t_{\mathrm{rf}} / N_{p}\right.$, filling all the rf buckets), $\epsilon \ll 1$ is usually guaranteed. However, $n_{r}$ (the harmonic number of an ERL, 343 for CBETA) can be a large number depending on the recirculation lattice, so $n_{r} \epsilon \ll 1$ is not guaranteed. This means the approximate formula needs to be applied with caution. Note that the top case in Eq. (13) corresponds to the $I_{\text {th }}$ in the trough region, and can be rewritten as:

$$
I_{\text {th }}=\frac{-2 c^{2}}{e(R / Q)_{\lambda} Q_{\lambda} \omega_{\lambda}} \frac{1}{T_{12} \sin \left(\omega_{\lambda} t_{r}\right)} .
$$

This formula has been derived in several literature regarding BBU [17-19]. Despite its limited applicability, the formula gives us insight on how to avoid a low $I_{\text {th }}$. Besides suppressing the HOM quality factor $Q_{\lambda}$, one can also adjust the recirculation time to avoid $\sin \left(\omega_{\lambda} t_{r}\right) \approx+1$ (or -1 ) when $T_{12}$ is negative (or positive). Theoretically $I_{\text {th }}$ can be infinite by making $T_{12}=0$. Unfortunately this can not be achieved in general with multiple cavities and $N_{p}>2$, since the $T_{12}$ between each pair of multipass cavities all needs to be zero. In reality the $T_{12}$ also depends on the length of the cavity, which will be discussed in Sec. V E. The strategies to improve the $I_{\text {th }}$ in general will be covered in Sec. V.

\section{B. One dipole-HOM with $N_{p}=4$}

In case $\mathrm{A}\left(N_{p}=2\right)$ we see that three analytic formulas exist: the general, linearized, and approximate formula. For a more general case with one dipole-HOM yet $N_{p}>2$, the general formula involves finding the maximum eigenvalue

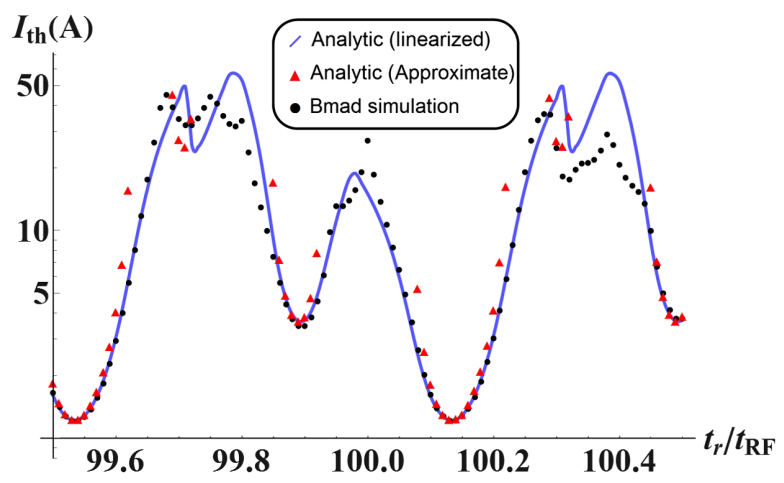

FIG. 15. Comparison of the $I_{\text {th }}$ obtained from the linearized formula and BMAD simulation for $N_{p}=4$. Parameters used are the same as in Fig. 14, with $T_{12}=T^{I J}$ and $t_{r}=t_{I}-t_{J}$ for any $I=J+1$. The trough regions are where the approximate formula (red triangles) is evaluated.

of a complex matrix [6]. Due to numerical difficulty we will not apply the general formula. Similar to Eq. (12), the linearized formula is [6]:

$D(\omega)=-\frac{\kappa}{2} \frac{1}{\left(\omega-\omega_{\lambda}\right) t_{b}+i \epsilon} \sum_{J=1}^{N_{p}} \sum_{I=J+1}^{N_{p}} e^{i \omega\left(t_{I}-t_{J}\right)} T^{I J}$,

in which $I$ and $J$ are the cavity pass index, $\left(t_{I}-t_{J}\right)$ is the recirculation time from pass $J$ to $I$, and $T^{I J}$ is the corresponding $T_{12}$ matrix element. To find the $I_{\text {th }}$ we again apply Eq. (11), and no complex matrix is involved. The approximate formula also exists, but works only for the "trough regions" in which $\sum_{J=1}^{N_{p}} \sum_{I=J+1}^{N_{p}} \sin \left[\omega\left(t_{I}-t_{J}\right)\right]$ $T^{I J} \leq 0$ :

$I_{\mathrm{th}}=\frac{-2 c^{2}}{e(R / Q)_{\lambda} Q_{\lambda} \omega_{\lambda}} \frac{1}{\sum_{J=1}^{N_{p}} \sum_{I=J+1}^{N_{p}} \sin \left[\omega_{\lambda}\left(t_{I}-t_{J}\right)\right] T^{I J}}$.

Figure 15 shows the comparison between BMAD simulation and the two analytic formulas. In contrast to the case with $N_{p}=2$ (Fig. 14), we now have three instead of one trough regions in one period. The number, depth, and location of the troughs depend on the signs and magnitudes of $T^{I J}$, or the optics of multiple recirculation passes. We again observe great agreement between simulation and the linearized formula at the trough regions, and the approximate formula agrees well only around the minimums.

\section{One dipole-HOM in two different cavities with $N_{p}=2$}

The complexity of this case comes from the interaction between the two HOMs via different recirculation passes. Figure 16 shows all the possible ways the HOMs excite themselves and each other. For example, the HOM of 


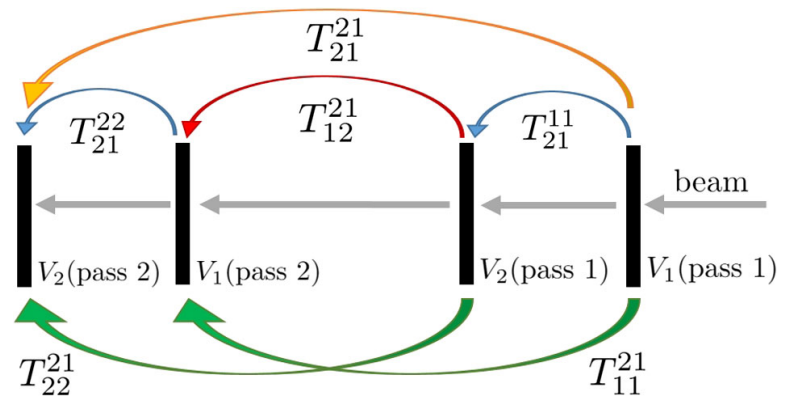

FIG. 16. Illustration of the case $\mathrm{C}$ configuration. $V_{j}$ denotes the HOM of cavity $j$, and $T_{i j}^{I J}$ is the $T_{12}$ from HOM $j$ of pass $J$ to HOM $i$ of pass $I$. Arrows with the same color indicate that the corresponding $T_{i j}^{I J}$ are assumed the same in order to derive Eq. (18).

cavity $1\left(V_{1}\right)$ can excite itself via recirculation (via the green arrow labeled $\left.T_{11}^{21}\right)$. It can also excite the HOM of cavity $2\left(V_{2}\right)$ in the same pass (via the blue arrows labeled $T_{21}^{11}$ for pass 1 and $T_{21}^{22}$ for pass 2).

Similar to Case B, the general formula involves calculating the eigenvalues of a complex matrix. However, the formula greatly simplifies if the two HOMs have identical characteristics, and the lattice has symmetric optics $\left(T_{21}^{22}=\right.$ $T_{21}^{11}$ and $T_{22}^{21}=T_{11}^{21}$ ) [6]:

$D(\omega)=-\frac{\kappa}{2} \frac{e^{i \omega t_{r}}\left[T_{11}^{21} \pm \sqrt{T_{12}^{21}\left(T_{21}^{21}+2 e^{-i \omega t_{r}} T_{21}^{11}\right)}\right]}{\left(\omega-\omega_{\lambda}\right) t_{b}+i \epsilon}$.

Comparing to Eq. (12) we see the equivalent $T_{12}$ becomes $\left(T_{11}^{21} \pm \sqrt{T_{12}^{21}\left(T_{21}^{21}+2 e^{-i \omega t_{r}} T_{21}^{11}\right)}\right)$, which has two possible values for a fixed $\omega$. Since Eq. (18) is a linearized formula, to find the $I_{\text {th }}$ we need to apply Eq. (11) while considering both values. In general one value gives a greater $\left|I_{0}^{-1}\right|$, which leads to the $I_{\text {th }}$. Equation (18) has several peculiarities which will be explained by the following three cases with special optics (See Table IV), and Fig. 17 shows the theory and simulation results for these cases.

In the case $\mathrm{C} 1, T_{12}^{21}=0$, which means that the second $\operatorname{HOM}(j=2)$ can not excite the first $\operatorname{HOM}(j=1)$. This is shown clearly by the red arrow in Fig. 16. Even though the first HOM can excite the second HOM in this case, there is no feedback from the second HOM. The two HOMs only feedback to themselves. The $I_{\text {th }}$ is therefore as large as that of with one single cavity only. Equation (18) supports this argument since the equivalent $T_{12}$ is now simply $T_{11}^{21}$, which

TABLE IV. The three subcases for case $\mathrm{C}$ with special optics.

\begin{tabular}{lc}
\hline \hline Case & Optics \\
\hline $\mathrm{C} 1$ & $T_{12}^{21}=0$ \\
$\mathrm{C} 2$ & $T_{21}^{22}=T_{21}^{11}=0$ \\
$\mathrm{C} 3$ & $T_{21}^{21}=0$ \\
\hline \hline
\end{tabular}

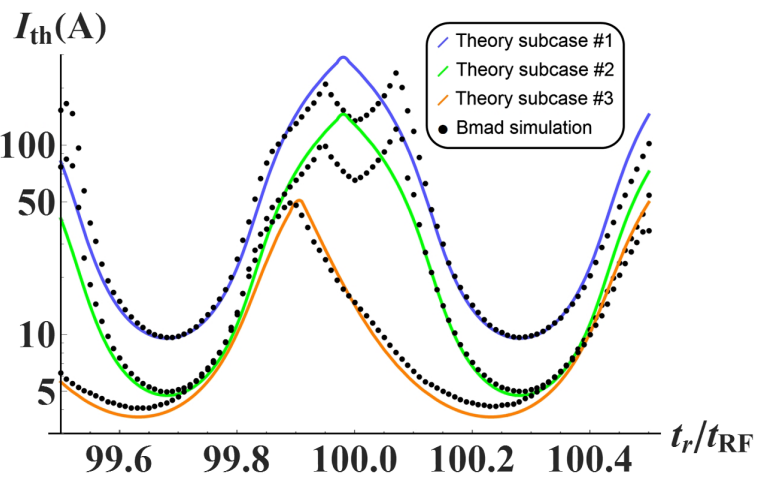

FIG. 17. Comparison of the $I_{\text {th }}$ obtained from the linearized formula and BMAD simulation for the case $\mathrm{C} 1$ (top curve), $\mathrm{C} 2$ (middle), and C3 (bottom). The HOM properties are the same as in case $\mathrm{A}$, and the optics are chosen carefully so $T_{11}^{21}=$ $-10 \mathrm{~m} /(1 \mathrm{GeV} / c)$ for all the three subcases.

agrees with Eq. (12) in the case A. The simulation results again agree well at the trough regions, as observed for all the linearized formulas before.

For the case $\mathrm{C} 2$, each HOM can still excite itself directly through $T_{i i}^{21}$ (the green arrows in Fig. 16). However, the two HOMs can now excite each other via recirculation through $T_{12}^{21}$ (the red arrow) and $T_{21}^{21}$ (the orange arrow). This mutual excitation results in extra feedback, and changes the equivalent $T_{12}$ to be $\left(T_{11}^{21} \pm \sqrt{T_{12}^{21} T_{21}^{21}}\right)$, which is independent of $\omega$. This means the $I_{\text {th }}$ occurs at the same $\omega$ as in the case $\mathrm{C} 1$, but the value is scaled down by a constant factor depending on $\sqrt{T_{12}^{21} T_{21}^{21}}$. The scaling effect is shown by the top two curves in Fig. 17. Note that if we swap the HOM index $i$ and $j$, the equivalent $T_{12}$ stays the same.

For the case $\mathrm{C} 3$ we have $T_{21}^{21}=0$. The two HOMs still excite other (via orange and blue arrows in Fig. 16), but not symmetrically as in the case $\mathrm{C} 2$. The bottom curve of Fig. 17 shows the corresponding $I_{\text {th }}$ profile, and the location of the trough regions clearly shifts from the two previous cases. This shift is expected due to the extra $e^{-i \omega t_{r}}$ term in Eq. (18). The crest regions might have vanished as we choose between the two quadratic values for greater $\left|I_{0}^{-1}\right|$. The choice at different $t_{r}$ varies with on the $e^{-i \omega t_{r}}$ term, which allows us to stay at the trough region given by one of the two values. The overall agreement with the simulation results also supports that the crest regions, at which linearized formula typically disagrees, have vanished.

\section{Two polarized dipole-HOMs in one cavity with $N_{p}=2$}

All the cases discussed so far assume that the HOMs are polarized in the horizontal direction only. With cylindrical symmetry there exists a vertical HOM for each horizontal $\mathrm{HOM}$, and the HOM pair has identical HOM characteristics except for the polarization angle. If the recirculation lattice has coupled beam optics between the two transverse phase 
spaces (i.e., nonzero $T_{14}$ and $T_{32}$ ), then the two HOMs could excite each other via recirculation. Similar to case A, we consider the simplest configuration with one cavity and $N_{p}=2$. For the case with $\epsilon \ll 1$ and $n_{r} \epsilon \ll 1$, the approximate formulas for the $I_{\text {th }}$ are [16]

$$
\begin{gathered}
I_{\mathrm{th}}=\min \left(I_{ \pm}\right), \\
I_{ \pm}= \begin{cases}-\frac{\epsilon}{\kappa} \frac{2}{T_{ \pm} \sin \left(\omega_{\lambda} t_{r}+\nu_{ \pm}\right)} & \text {if it is }<0 \\
\frac{2}{\kappa T_{ \pm}} \sqrt{\epsilon^{2}+\left(\frac{t_{b}}{t_{r}}\right)^{2} \times \operatorname{minmod}\left(\omega_{\lambda} t_{r}+\nu_{ \pm}, \pi\right)} & \mathrm{o} / \mathrm{w},\end{cases}
\end{gathered}
$$

$$
\begin{gathered}
T_{ \pm} e^{i \nu_{ \pm}}=\frac{T_{12}+T_{34}}{2} \pm \sqrt{\left(\frac{T_{12}-T_{34}}{2}\right)^{2}+T_{14} T_{32}} \\
\text { with } T_{ \pm}, \nu_{ \pm} \in \Re \text { and } T_{ \pm}>0 .
\end{gathered}
$$

Note that Eq. (19) is essentially Eq. (13) with $T_{12}$ replaced by $T_{ \pm}$, and $\nu_{ \pm}$added to $\omega_{\lambda} t_{r}$. From Eq. (19) we see there are two candidates $\left(I_{+}\right.$and $\left.I_{-}\right)$for the $I_{\text {th }}$, and the nature of coupling (i.e., the matrix elements in Eq. (21) determines which one is the $I_{\text {th }}$ at different $t_{r}$ ). We define $\Delta \nu=\left|\nu_{+}-\nu_{-}\right|$, which measures the phase shift between $I_{+}\left(t_{r}\right)$ and $I_{-}\left(t_{r}\right)$. To compare the formula with simulation results, we again focus on three cases with specified optics, listed in Table $\mathrm{V}$ below.

Figure 18 compares the $I_{\text {th }}$ obtained from Eq. (19) and BMAD simulation for the case D1. To study the behavior of coupling, the two candidates $I_{ \pm}\left(t_{r}\right)$ are also plotted. Note that both $I_{ \pm}\left(t_{r}\right)$ curves have distinct crest and trough regions as in case A. The two curves are $\Delta \nu=\pi$ out of phase, causing the $I_{\text {th }}$ to always stay at the trough regions. This is expected for two reasons. First, the lattice has no coupling $\left(T_{14}=T_{32}=0\right)$, so the two HOMs do not excite each other. Mathematically we see $T_{+}=\left|T_{12}\right|$ and $T_{-}=\left|T_{34}\right|$. The second reason is about the physical difference between the trough and crest region. The trough region has lower $I_{\text {th }}$ because a particle with positive x offset receives positive kick in $\mathrm{x}$ after recirculation. In the crest region the particle instead receives a negative kick in $\mathrm{x}$, resulting in a more tolerable $I_{\text {th }}$. Since we have $T_{12}=-T_{34}$ for subcase 1 , when $\mathrm{x}$ motion benefits from the crest region,

TABLE V. The three subcases for case D with specified optics. We set $x=-10 \mathrm{~m} /(\sqrt{2} \mathrm{GeV} / c)$, and the rest of the matrix elements are set to meet symplecticity, consistent with [16]. The optics for case D3 was specifically chosen to obtain $\Delta \nu=2 \pi$.

\begin{tabular}{lcccccc}
\hline \hline Case & $T_{12}$ & $T_{14}$ & $T_{32}$ & $T_{34}$ & $T_{-} / T_{+}$ & $\Delta \nu$ \\
\hline D1 & $x$ & 0 & 0 & $-x$ & 1 & $\pi$ \\
D2 & $x$ & $3 x$ & $-2 x$ & $4 x$ & 1 & 4.97 \\
D3 & $x$ & $(2+\sqrt{6}) x$ & $(-2+\sqrt{6}) x$ & $3 x$ & 13.9 & $2 \pi$ \\
\hline \hline
\end{tabular}

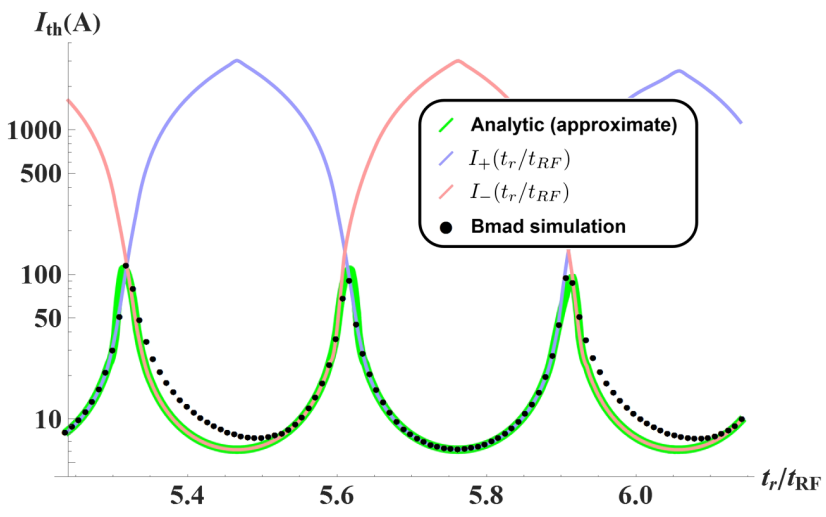

FIG. 18. Comparison of the $I_{\text {th }}$ obtained from the approximate analytic formula [Eq. (19)] and BMAD simulation for case D1. The two candidates for $I_{\text {th }}\left[I_{ \pm}\right.$from Eq. (20)] are also plotted. Parameters: $t_{b}=t_{\mathrm{rf}}=1 / 1.3 \mathrm{GHz}, \quad \omega_{1}=\omega_{2}=2 \pi \times 2.2 \mathrm{GHz}$, $Q_{1}=Q_{2}=100,(R / Q)_{1}=(R / Q)_{2}=10^{4} \Omega$.

$y$ motion suffers from the positive feedback at the trough region, and vice versa. The $I_{\text {th }}$ occurs when either x or y motion becomes unstable, not both. If we instead had $T_{12}=T_{34}$, the two candidate curves will overlap each other (in phase with equal magnitude), indicating that $\mathrm{x}$ and $\mathrm{y}$ motion are identical. In other words, without optical coupling the $I_{\text {th }}$ either follows Fig. 14 (with distinct crest and trough regions) or Fig. 18 (with trough regions only). The BMAD simulation agrees with the approximate formula well, especially in the trough regions of $I_{+}\left(t_{r}\right)$. Reasons for the slight overestimate of $I_{-}\left(t_{r}\right)$ at the crest region are to be investigated.

Figure 19 shows the comparison for the second subcase. The $I_{\text {th }}$ for this particular set of optics has been checked in [16] for a specific $t_{r}$ value, and here we check against various $t_{r}$ values with BMAD simulation. Similar to the case D1, case D2 has $T_{+}=T_{-}$, but the different value of $\Delta \nu$ drastically changes the $I_{\text {th }}$ behavior at different $t_{r}$. Since $\Delta \nu \neq \pi$, the crest regions of the two candidates partially overlap, giving a peak region to the $I_{\text {th }}$ curve.

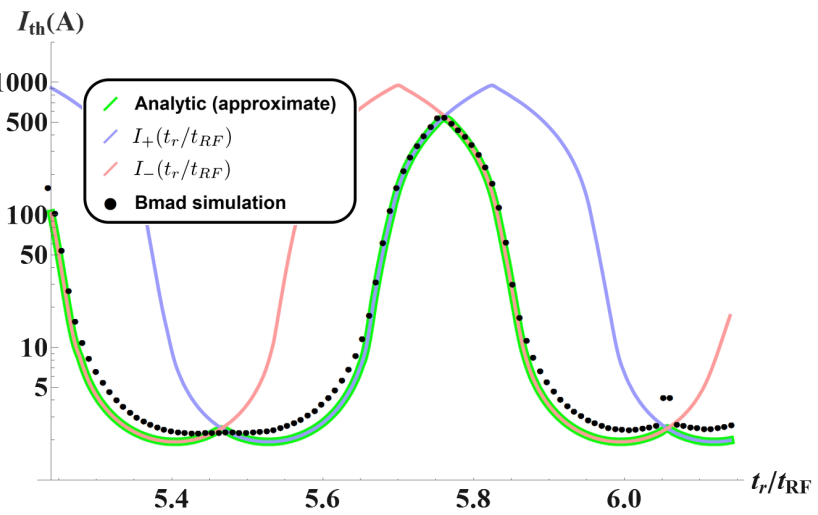

FIG. 19. Comparison of the $I_{\text {th }}$ obtained from the approximate analytic formula [Eq. (19)], the two candidates [Eq. (20)], and the BMAD simulation for the case D2. The parameters used are identical as in the case D1, except for the optics. 


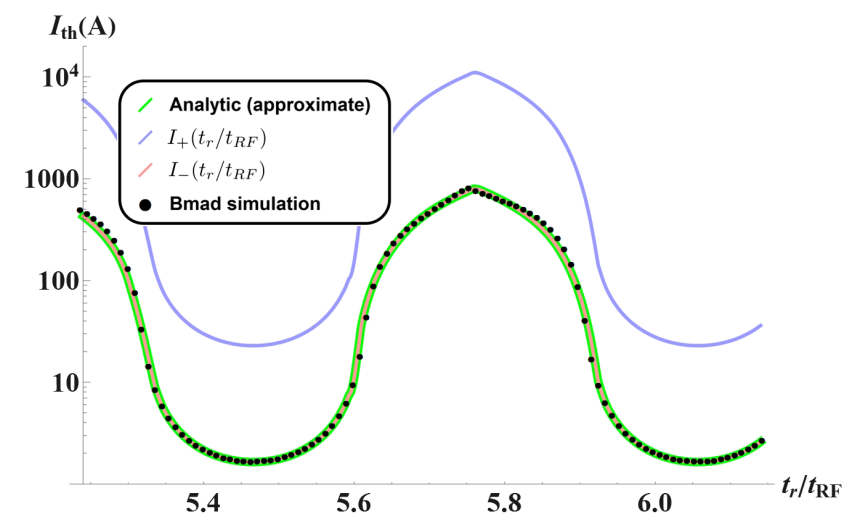

FIG. 20. Comparison of the $I_{\text {th }}$ obtained from the approximate analytic formula [Eq. (19)], the two candidates [Eq. (20)], and the BMAD simulation for the case D3. The parameters used are identical as in the case D1, except for the optics.

Since coupling exists now, the two transverse motions affect each other, and should not be treated independently. Around the peak, the motions together benefit from the crest regions, resulting in a greater $I_{\text {th }}$. Again, BMAD simulation agrees well with the approximate formula.

Lastly, Fig. 20 shows the comparison for the case D3. The optics are carefully chosen such that $\Delta \nu$ is $2 \pi$, or equivalently zero. This causes the two candidate curves to be in phase, and the ratio $I_{+}\left(t_{r}\right) / I_{-}\left(t_{r}\right)=T_{-} / T_{+}$remains constant. The $I_{\text {th }}$ curve will always follow the "smaller" candidate curve $\left(I_{-}\left(t_{r}\right)\right.$ with our choice of optics). Recall that in the case D1 the two candidate curves would overlap (and be in phase) if $T_{12}$ and $T_{34}$ have the same sign. One might thus wonder what is the effect of coupling in the case D3. In contrast to the case D2 in which coupling changes both the magnitude and phase of the two curves, coupling here only changes their magnitude. The $I_{\text {th }}$ magnitude therefore entirely increases or decreases at all $t_{r}$ depending on the beam optics.

The three cases above have shown that optical coupling can strongly affect the $I_{\text {th }}$. However, in reality it can be difficult to achieve specific optics in order to reach a high $I_{\text {th }}$. For a more general case in BBU with more HOMs and $N_{p}>2$, neither the linearized formula nor the approximate formula exists. The general formula becomes more difficult to apply numerically, so we rely on simulation to find the $I_{\text {th }}$. The agreement with the analytic formulas in all the example cases makes us confident to use BMAD to calculate the $I_{\text {th }}$ of CBETA.

\section{E. Comment on recirculation $T_{12}$}

Let us refocus on the most elementary BBU case with one HOM and $N_{p}=2$ (Case A). Since the BBU theory derived in [6] assumes a thin-lens cavity, the $T_{12}$ in the formulas corresponds to the $T_{12}$ of the recirculation beamline. This is however an approximation to the reality since particles undergo transverse motion through a cavity with

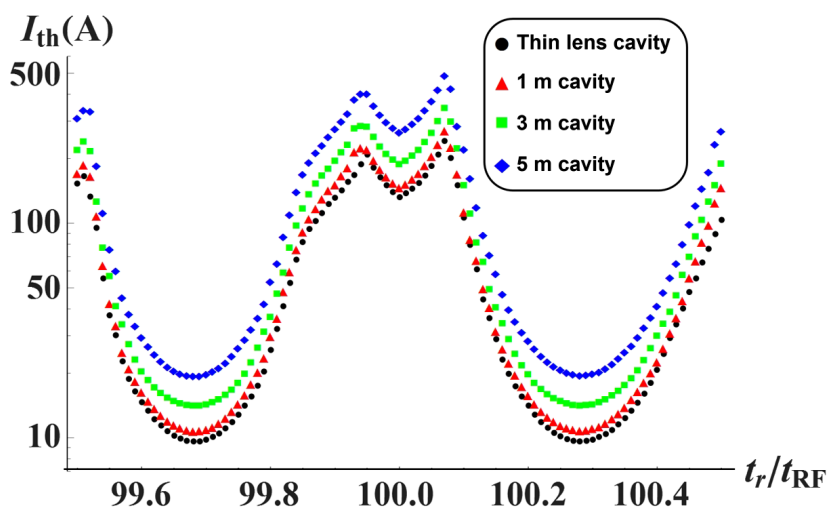

FIG. 21. Comparison of the $I_{\text {th }}$ obtained from the BMAD simulation for case A with different cavity length. Parameters used are identical as in Fig. 14.

nonzero length. Consequently the equivalent $T_{12}$ would depend on other matrix elements $\left(T_{11}, T_{21}, T_{22}\right.$, etc.) of the recirculation beamline, as well as the transfer matrix of the cavity itself. This effect is included in BMAD simulation, with the cavity transfer matrix derived in [20], and the transverse HOM kick given instantly at the center of the cavity. Figure 21 shows the $I_{\text {th }}$ for case A with varying cavity length. The optics of the recirculation beam line is held constant. In our case, increasing cavity length lowers the equivalent $\left|T_{12}\right|$, resulting in a greater $I_{\text {th }}$ for all $t_{r}$. Physically this reflects the transverse focusing effect of the cavity.

In reality the HOM kick is not instant at a specific point of the cavity, but gradual depending the time varying HOM field. A more realistic simulation would therefore integrate the field contribution from both the fundamental mode and the HOM to calculate the exact particle trajectory through the cavity. Since the HOM field depends on the interaction history of the traversed beam, the simulation can be computationally intensive.

\section{SCALING OF MULTIPASS $\boldsymbol{I}_{\text {th }}$ WITH $\boldsymbol{N}_{r}$}

The scaling of $I_{\text {th }}$ over the number of linac pass $N_{p}$ was estimated to be

$$
I_{\text {th }}\left(N_{r}\right) \approx \frac{1}{N_{r}\left(2 N_{r}-1\right)} I_{\text {th }}(1)
$$

in [6] for multiturn accelerators with $N_{p}=2 N_{r}$. Here we revisit the scaling law and focus on the case with symmetric ERLs. Symmetry means that the optics and time of flights of the accelerating passes are mirror symmetric to those of the decelerating passes. Assuming there is only one dipole $\mathrm{HOM}$, the formula for the multi-pass $I_{\text {th }}$ can be written as, from the BBU theory:

$$
I_{\mathrm{th}}=\frac{2 \epsilon}{\kappa} \frac{1}{\max _{\omega} \Sigma S}, \quad \Sigma S>0 .
$$




$$
\Sigma S=-\sum_{J=1}^{N_{p}} \sum_{I=J+1}^{N_{p}} \sin \left[\omega\left(t_{I}-t_{J}\right)\right] \sqrt{\frac{\beta_{I} \beta_{J}}{p_{I} p_{J}}} \sin \left(\phi_{I J}\right),
$$

in which $\beta_{I}$ and $p_{I}$ are the beta function and momentum at pass $I$, and $\phi_{I J}$ is the phase advance from pass $J$ to pass $I$. For an ERL we have the following constraints:

$$
\begin{gathered}
N_{p} \text { is even. } \\
\left(t_{I+1}-t_{I}\right)= \begin{cases}\left(m_{I}+\frac{1}{2}\right) \times t_{\mathrm{rf}} & \text { if } I=N_{p} / 2 \\
m_{I} t_{\mathrm{rf}} & \text { otherwise }\left(m_{I} \in \mathbb{N}\right)\end{cases}
\end{gathered}
$$

For a symmetric ERL we have additional constraints:

$$
\begin{gathered}
t_{I}+t_{N_{p}-I+1}=\text { const } \forall I . \\
\beta_{I}=\beta_{N p-I+1} \forall I . \\
p_{I}=p_{N p-I+1} \forall I . \\
\phi_{I J}=\phi_{\left(N_{p}-J+1\right)\left(N_{p}-I+1\right)} \forall I>J .
\end{gathered}
$$

The goal here is to obtain the scaling law of the minimum $I_{\text {th }}$ for a symmetric ERL with adjustable phase advances $\phi_{I J}$ and fixed $t_{I}, \beta_{I}$, and $p_{I}$. We define:

$$
\min \left[I_{\mathrm{th}}\right]=\frac{2 \epsilon}{\kappa}\left(\max _{\omega, \phi_{I J}} \Sigma S\right)^{-1}, \quad \Sigma S>0 .
$$

For simplicity we choose $\sqrt{\beta_{I} / p_{I}}=$ constant. With $\sigma_{x} \approx \sqrt{\beta_{x} \epsilon_{n} /(\beta \gamma)}$, this means the beams size is the same for all $I$. The symmetry constraint on $\phi_{I J}$ reduces the number of free phases from $N_{p}\left(N_{p}-1\right) / 2$ to $N_{p} / 2$. In general, each term in Eq. (31) can be positive or negative depending on the values of $\left(\omega, \phi_{I J}\right)$, and the minimum $I_{\text {th }}$ occurs when the sum of these terms, neglecting the minus sign at the front, is the most negative. Figure 22 below shows the resultant $\min \left[I_{\text {th }}\right]$ with different $N_{p}$ and $\omega_{\lambda}$ after optimization using Mathematica.

With no prediction it turns out that the minimum $I_{\text {th }}$ closely follow a new scaling law:

$$
\min \left[I_{\mathrm{th}}\left(N_{r}\right)\right] \approx\left(\frac{1}{N_{r}}\right)^{2} \min \left[I_{\mathrm{th}}(1)\right]
$$

Instead of the estimated value of $1 / N_{r}\left(2 N_{r}-1\right)$ in [6], the scaling factor for the $\min \left[I_{\mathrm{th}}\right]$ for a symmetric ERL is found to be just $\left(1 / N_{r}\right)^{2}$. While the exact scaling factor requires rigorous mathematical proof, the new scaling factor provides more insight into the $I_{\text {th }}$ scaling for symmetric ERLs.

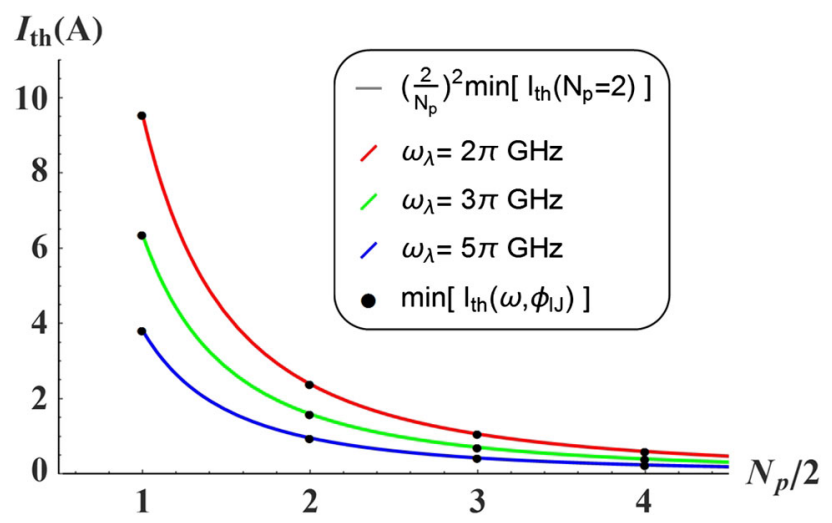

FIG. 22. Scaling of the $\min \left[I_{\text {th }}\right]$ over $N_{p}$ for symmetric ERLs with different $\omega_{\lambda}$. Parameters: $c t_{\mathrm{rf}}=0.5 \mathrm{~m}, t_{b}=t_{\mathrm{rf}}, Q_{\lambda}=100$, $(R / Q)_{\lambda}=10^{4} \Omega, t_{I+1}-t_{I}=100 t_{\mathrm{rf}}$ (with an additional $\frac{1}{2} t_{\mathrm{rf}}$ for $\left.I=N_{p} / 2\right), \beta_{I}=10 \mathrm{~m}$, and $p_{I} \approx 1 \mathrm{GeV} / c$ for all $I$.

Note that the previous factor in [6] was estimated to be $1 / N_{r}\left(2 N_{r}-1\right)$ because there are $N_{r}\left(2 N_{r}-1\right)$ terms in Eq. (23). However, due to the symmetry constraints, the number of distinct terms is reduced to $N_{r}^{2}$, which happens to correspond to the new scaling factor of $\left(1 / N_{r}\right)^{2}$. While the previous factor was obtained assuming that the multipass $T_{I J}$ s have very similar values for all $I>J$, the new scaling factor assumes mirror symmetrical optics. In a realistic ERL like CBETA, the former assumption is more difficult to achieve than the latter one, making the new scaling law more relevant. Since ERLs are usually designed to achieve a high $I_{\text {th }}$ instead of the minimum $I_{\text {th }}$, the applicability of the new scaling law might first seem limited. However, with many cavities and HOMs, the $I_{\text {th }}$ tends to locate at the trough region, with a value close to the minimum $I_{\text {th }}$. The new scaling law for the minimum $I_{\text {th }}$ can therefore serve as a decent approximation for the scaling of the actual $I_{\text {th }}$.

\section{EFFECT OF CHROMATICITY ON BBU $\boldsymbol{I}_{\text {th }}$}

When phase space filamentation is relevant, e.g., when the chromaticity $(\xi=d \nu / d \delta)$ times the energy spread $\left(\sigma_{\delta}\right)$ is of order 1, the BBU instability can be suppressed [15]. Here we estimate this effect on the effective $T_{12}$ in the BBU theory. The $T_{12}$ seen by the design particle can be written as:

$$
T_{12}(\delta=0)=\sqrt{\frac{\beta_{0} \beta_{1}}{p_{0} p_{1}}} \sin \left(2 \pi \nu_{0}\right),
$$

in which $\beta_{0}\left(\beta_{1}\right)$ and $p_{0}\left(p_{1}\right)$ are the beta function and momentum at the beginning (end) of the recirculation arc around the cavity, and $2 \pi \nu_{0}$ is the phase advance of the design particle.

For a particle with relative energy deviation $\delta$, the phase advance is $2 \pi\left(\nu_{0}+\xi \delta\right)$. Let $\rho(\delta)$ denote the $\delta$ distribution of the beam, then the effective $T_{12}$ can be written as: 


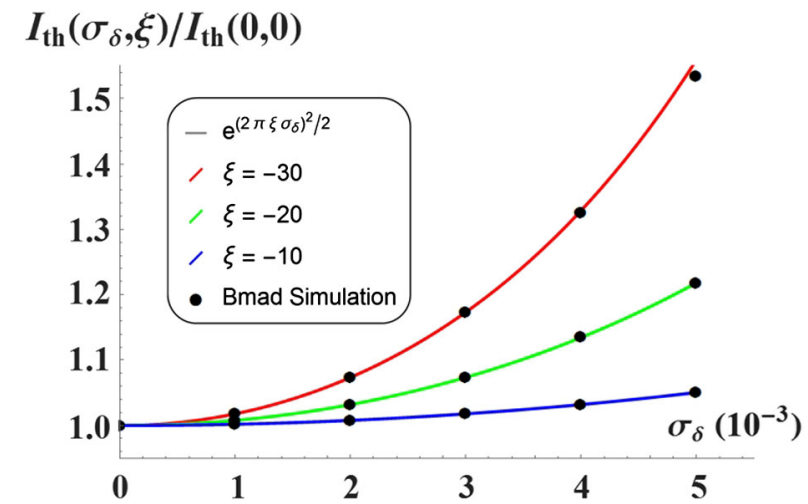

FIG. 23. The $I_{\text {th }}$ results from tracking a Gaussian beam (2000 particles per bunch) through a "one-HOM, $N_{p}=2$ " lattice with different $\left(\sigma_{\delta}, \xi\right)$. The three curves correspond to the factor $e^{-\left(2 \pi \xi \sigma_{\delta}\right)^{2} / 2}$ with different $\xi$, and the black dots are the simulation results. Parameters: same as in case A (See Fig. 14).

$$
\hat{T}_{12}=\sqrt{\frac{\beta_{0} \beta_{1}}{p_{0} p_{1}}} \int_{-\infty}^{\infty} \rho(\delta) \sin [2 \pi(\nu+\xi \delta)] d \delta
$$

Assume $\rho(\delta)$ is a Gaussian distribution with $(\mu, \sigma)=$ $\left(0, \sigma_{\delta}\right)$, and apply the identity $\sin (A+B)=\sin (A)$ $\cos (B)+\cos (A) \sin (B)$. The second term in the integral then vanishes (odd in $\delta$ ), yielding:

$$
\begin{aligned}
\hat{T}_{12} & =\sqrt{\frac{\beta_{0} \beta_{1}}{p_{0} p_{1}}} \frac{\sin \left(2 \pi \nu_{0}\right)}{\sqrt{2 \pi \sigma_{\delta}^{2}}} \int_{-\infty}^{\infty} e^{-\delta^{2} / 2 \sigma_{\delta}^{2}} \cos (2 \pi \xi \delta) d \delta \\
& =T_{12}(\delta=0) \frac{1}{\sqrt{2 \pi \sigma_{\delta}^{2}}}\left(\sqrt{2 \pi \sigma_{\delta}^{2}} e^{-(2 \pi \xi)^{2} \sigma_{\delta}^{2} / 2}\right) \\
& =T_{12}(\delta=0) e^{-\left(2 \pi \xi \sigma_{\delta}\right)^{2} / 2}
\end{aligned}
$$

Figure 23 below shows the $I_{\text {th }}$ results of tracking a Gaussian beam (with different $\sigma_{\delta}$ ) through a "one-HOM, $N_{p}=2$ " lattice (with different chromaticity $\xi$ ). From the most elementary BBU case, we know $I_{\text {th }} \propto T_{12}^{-1}$, so from the chromaticity analysis above we expect:

$$
I_{\text {th }}\left(\sigma_{\delta}, \xi\right) / I_{\text {th }}(0,0)=e^{-\left(2 \pi \xi \sigma_{\delta}\right)^{2} / 2} .
$$

Bmad simulation results agree with this prediction for various $\left(\sigma_{\delta}, \xi\right)$.

Since the factor $e^{-\left(2 \pi \xi \sigma_{\delta}\right)^{2} / 2}$ is smaller than unity, the effective $T_{12}$ becomes smaller in magnitude, resulting in a greater $I_{\text {th }}$ all the time. For a multipass lattice with multiple cavities, the effective $T_{12}$ elements scale up differently depending on the chromaticity of the return loops. Assuming that $\delta$ distribution remains Gaussian-like, the $I_{\text {th }}$ is expected to be greater than that with only the design particle.

CBETA has no sextupoles, and the natural horizontal chromaticity $\xi$ of its four loops is approximately, from the
$I_{\mathrm{th}}\left(\sigma_{\delta}\right) / I_{\mathrm{th}}(\mathbf{0})$

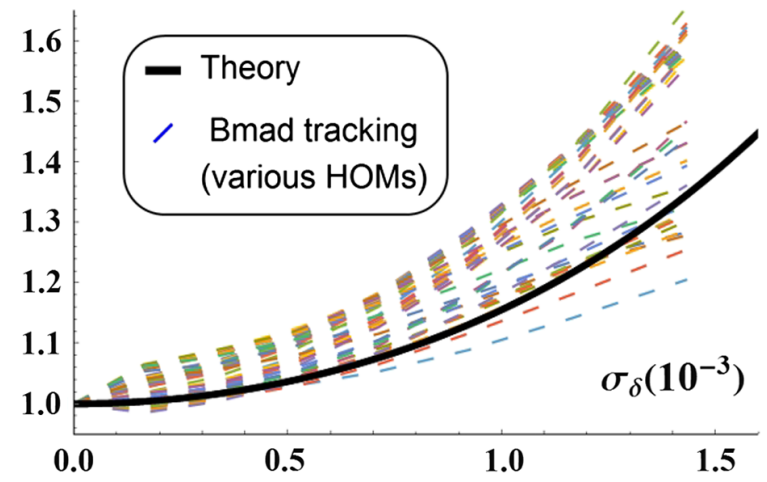

FIG. 24. Fifty $I_{\text {th }}$ results from tracking a beam (3000 particles per bunch) through the CBETA 1-pass lattice with different $\sigma_{\delta}$ and HOM assignments. Each of the 50 dashed curves corresponds to one specific HOM assignment, and the thick curve is the theory prediction with a Gaussian beam.

lowest to the highest design energy, $-85.6,-25.3,-16.5$, and -14.9 . With an energy spread $\sigma_{\delta}$ of $10^{-3}$, the effective $T_{12}$ elements of the lowest energy loop decreases by $13.4 \%$. This means that the $I_{\text {th }}$ of CBETA 1-pass mode could increase up to $15.5 \%$ assuming only one dipole HOM exists. To check this we tracked a beam with 3000 particles per bunch through the CBETA 1-pass lattice with varying $\sigma_{\delta}$ and different HOM assignments. Note that these BBU simulations with multiple particles per bunch are computationally intensive comparing to the cases with single particle tracking.

Figure 24 shows that for 50 different HOM assignments, the $I_{\text {th }}$ increases as $\sigma_{\delta}$ increases, and the result curves follow relatively close to the theory prediction. The discrepancy in some of the curves is likely due to the non-Gaussian distribution of the accelerated bunches. Regardless, the results indicate that CBETA 1-pass can reach a higher $I_{\text {th }}$ than predicted with single particle tracking. Moreover, the theory can be used to approximate the case non-Gaussian bunches. Although increasing the energy spread seems to help raising the $I_{\text {th }}$, it will eventually cause particle loss due to lattice dispersion. A large energy spread can also cause undesired ERL operation at the cavities. The limit and reliability of this method to increase the $I_{\text {th }}$ therefore requires experimental testing. For the 4-pass mode, the chromaticity magnitudes of the higher energy loops are much smaller, giving a small chance to increase the $I_{\text {th }}$.

\section{CONCLUSION}

To establish the trustworthiness of BBU simulations for CBETA, agreement has been found between the BBU theory and BMAD simulations for multiturn ERL setups. This gives us confidence in BMAD simulation for determining the $I_{\text {th }}$ for ERL lattices with multipass cavities and multiple HOMs, like CBETA. Simulation results show that 
for CBETA (both the 1-pass and 4-pass mode), the $I_{\text {th }}$ can always surpass the low design current of $1 \mathrm{~mA}$, and can reach the high goal of $40 \mathrm{~mA}$ in over $98 \%$ of the cases with realistic cavity construction errors.

In the remaining $2 \%$ of the cases, the $I_{\text {th }}$ can be improved by adjusting the lattice optics, which has been an effective method for 1-turn ERLs like the Jefferson FEL-ERL. Varying phase advances is shown to be more effective than introducing $\mathrm{x}-\mathrm{y}$ coupling. Also, both methods are shown less effective in multiturn ERLs than in the 1-turn arrangement. It is intended to verify these observations experimentally, and it will be interesting to test the applicability and effectiveness of all these methods in CBETA.

The scaling law of the $I_{\text {th }}$ with $N_{r}$ is revisited for the case with symmetric ERLs, and the scaling factor is found to be approximately $1 / N_{r}^{2}$. This new scaling law has better applicability for ERLs than the previously approximated factor of $1 / N_{r}\left(2 N_{r}-1\right)$ in [6]. Also, the effect of lattice chromaticity on the $I_{\text {th }}$ is studied assuming bunches with an Gaussian energy spread, and a relationship between the $I_{\text {th }}$, chromaticity, and energy spread was found. Results with the CBETA 1-pass lattice show that the $I_{\text {th }}$ in reality can be higher than predicted using single particle tracking.

\section{ACKNOWLEDGMENTS}

We acknowledge Nicholas Valles for the HOM spectrum calculation and David Sagan for assistance with Bmad simulation. This work was performed with the support of NYSERDA (New York State Energy Research and Development Agency).

[1] G. H. Hoffstaetter, D. Trbojevic, C. Mayes, N. Banerjee, J. Barley, I. Bazarov, A. Bartnik, J. S. Berg, S. Brooks, D. Burke et al., CBETA Design Report, Cornell-BNL ERL Test Accelerator, arXiv:1706.04245.

[2] Proceedings, Workshop to Explore Physics Opportunities with Intense, Polarized Electron Beams up to $300 \mathrm{MeV}$, edited by R. Milner, Roger Carlini, and Frank Maas (AIP, Cambridge, USA, 2013).

[3] D. Androic et al. (Qweak Collaboration), First Determination of the Weak Charge of the Proton, Phys. Rev. Lett. 111, 141803 (2013).

[4] F. Albert, S. G. Anderson, D. J. Gibson, R. A. Marsh, S. S. Wu, C. W. Siders, C.P. J. Barty, and F. V. Hartemann, Design of narrow-band Compton scattering sources for nuclear resonance fluorescence, Phys. Rev. ST Accel. Beams 14, 050703 (2011).

[5] D. Trbojevic, S. Bellavia, M. Blaskiewicz, S. Brooks, K. Brown, C. Liu, W. Fischer, C. Franck, Y. Hao, G. Mahler et al., CBETA - Cornell University Brookhaven National Laboratory Electron Energy Recovery Test Accelerator, in Proceedings, 8th International Particle Accelerator Conference (IPAC2017): Copenhagen, Denmark, 2017,
TUOCB3, http://accelconf.web.cern.ch/AccelConf/ ipac2017/papers/tuocb3.pdf.

[6] G. H. Hoffstaetter and I. V. Bazarov, Beam-breakup instability theory for energy recovery linacs, Phys. Rev. Accel. Beams 7, 054401 (2004).

[7] D. Sagan, Bmad Simulation Software, https://www.classe .cornell.edu/bmad/.

[8] N. Valles, Ph.D., Cornell University, 2014, https://www .classe.cornell.edu/rsrc/Home/Research/GradTheses/ Valles_Nicholas.pdf.

[9] D. G. Myakishev and V. P. Yakovlev, CLANS2 - a code for calculation of multipole modes in axisymmetric cavities with absorber ferrites, Proceedings of the 18th Particle Accelerator Conference, New York, 1999 (IEEE, New York, 1999), pp. 2775-2777.

[10] W. Lou and G. H. Hoffstaetter, Beam-breakup studies for the 4-pass Cornell-Brookhaven Energy-Recovery Linac Test Accelerator, in Proceedings, 9th International Particle Accelerator Conference (IPAC2018): Vancouver, Canada, 2018, THPAF022, https://accelconf.web.cern .ch/AccelConf/ipac2018/papers/thpaf022.pdf.

[11] R. Rand and T. Smith, Beam optical control of beam breakup in a recirculating electron accelerator, Part. Accel. 2, 1 (1980).

[12] C. D. Tennant, K. B. Beard, D. R. Douglas, K. C. Jordan, L. Merminga, E. G. Pozdeyev, and T. I. Smith, First observations and suppression of multipass, multibunch beam breakup in the Jefferson Laboratory free electron laser upgrade, Phys. Rev. Accel. Beams 8, 074403 (2005).

[13] D. R. Douglas, K. C. Jordan, L. Merminga, E. G. Pozdeyev, C. D. Tennant, H. Wang, T. I. Smith, S. S. Simrock, I. V. Bazarov, and G. H. Hoffstaetter, Experimental investigation of multibunch, multipass beam breakup in the Jefferson Laboratory Free Electron Laser Upgrade Driver, Phys. Rev. Accel. Beams 9, 064403 (2006).

[14] R. Kazimi et al., Observation and mitigation of multipass $\mathrm{BBU}$ in CEBAF, in Proceedings of the 11th European Particle Accelerator Conference, Genoa, 2008 (EPS-AG, Genoa, Italy, 2008), WEPP087.

[15] V. N. Litvinenko, Chromaticity of the lattice and beam stability in energy recovery linacs, Phys. Rev. Accel. Beams 15, 074401 (2012).

[16] G. H. Hoffstaetter, I. V. Bazarov, and C. Song, Recirculating beam-breakup thresholds for polarized higher-order modes with optical coupling, Phys. Rev. Accel. Beams 10, 044401 (2007).

[17] V. Volkov, J. Knobloch, and A. Matveenko, Beam breakup instability suppression in multicell superconducting $\mathrm{rf}$ guns, Phys. Rev. Accel. Beams 14, 054202 (2011).

[18] B. C. Yunn, Expressions for the threshold current of multipass beam breakup in recirculating linacs from single cavity models, Phys. Rev. Accel. Beams 8, 104401 (2005).

[19] E. Pozdeyev, Regenerative multipass beam breakup in two dimensions, Phys. Rev. Accel. Beams 8, 054401 (2005).

[20] J. Rosenzweig and L. Serafini, Transverse particle motion in radio-frequency linear accelerators, Phys. Rev. E 49, 1599 (1994). 